Airborne Astronomy Program Medium Altitude Missions Branch Preprint Series 061

$$
\begin{gathered}
\text { Go } 0.37 \\
10-37 \\
p-72
\end{gathered}
$$

\title{
The Abundance and Distribution of Water Vapor in Jupiter's Atmosphere
}

Gordon L. Bjoraker, Harold P. Larson, and Virgil G. Kunde

\section{(BASA-TM-88366) THE AEUILANCE AND} CISTRIBUTION OF FATER VAPCR IN JUEITER'S

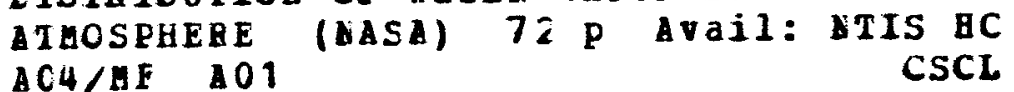
$\triangle O 4 / \triangle F$ D 01 $\begin{array}{ll}034 & \text { Unclas } \\ \text { H } 1 / 89 & \text { Co7c059 }\end{array}$

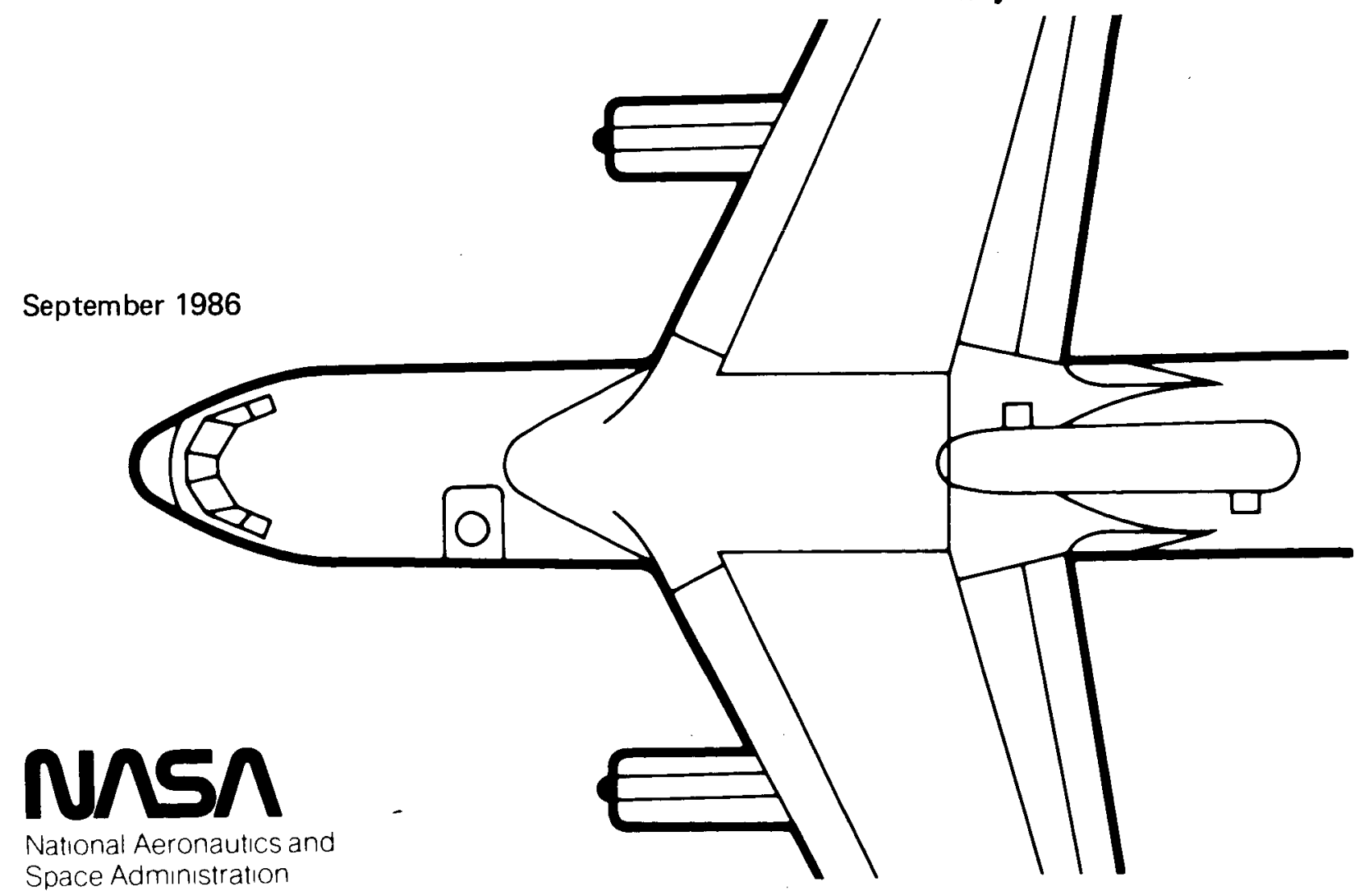




\section{The Abundance and Distribution of Water Vapor in Jupiter's Atmosphere}

Gordon L. Bjoraker,

Harold P. Larson, Lunar and Planetary Laboratory, University of Arizona, Tucson, Arizona Virgil G. Kunde, Laboratory for Extraterrestrial Physics, Goddard Space Flight Center,

Greenbelt, Maryland

September 1986

\section{N/Sก}

National Aeronautics and

Space Administration

Ames Research Center

Moffett Field, California 94035 


\section{ABSTRACT}

The atmospheric transmission window between 1800 and $2250 \mathrm{~cm}^{-1}$ in Jupiter's atmosphere was observed from the Kuiper Airborne Observatory (KAO) and by the infrared spectrometer (IRIS) on Voyager. The vertical distribution of $\mathrm{H}_{2} \mathrm{O}$ was derived for the 1 to 6 bar portion of Jupiter's troposphere. The spatial variation of $\mathrm{H}_{2} \mathrm{O}$ was measured using IRIS spectra of the Hot Spots in the North and South Equatorial Belts, the Equatorial Zone, and for an average of the North and South Troplcal Zones. The $\mathrm{H}_{2} \mathrm{O}$ column abundance above the 4 bar level is the same in the zones as In the SEB Hot Spots, about $20 \mathrm{~cm}$-amagat. The NEB Hot Spots are desiccated by a factor of 3 with respect to the rest of Jupiter. For an average between -40 to $40^{\circ}$ latitude, the $\mathrm{H}_{2} \mathrm{O}$ mole fraction, $\mathrm{qH}_{2} \mathrm{O}$, is saturated for $\mathrm{p}<2$ bars, $\mathrm{qH}_{2} \mathrm{O}=4 \times 10^{-6}$ in the 2 to 4 bar range and it increases to $3 \times 10^{-5}$ at 6 bars. A similar vertical profile applies to the spatially resolved zone and belt spectra, except that $\mathrm{H}_{2} \mathrm{O}$ falls off more rapidly at $\mathrm{P}<4$ bars in the NEB Hot Spots. The massive $\mathrm{H}_{2} \mathrm{O}$ cloud at 5 bars, $\mathrm{T}=273 \mathrm{~K}$, proposed in solar composition models, is inconsistent with the observations. Instead, a thin $\mathrm{H}_{2} \mathrm{O}$ ice cloud would form at 2 bars, $\mathrm{T}=200 \mathrm{~K}$. The $0 / \mathrm{H}$ ratio in Jupiter, inferred from $\mathrm{H}_{2} \mathrm{O}$ measurements in both belts and zones at 6 bars, is depleted by a factor of 50 
with respect to the Sun. The implications for the origin of Jupiter of globally depleted $0 / \mathrm{H}$, but enhanced $\mathrm{C} / \mathrm{H}$ and $\mathrm{N} / \mathrm{H}$, are discussed.

Subject headings : infrared : spectra -- planets : abundances -- planets : atmospheres -- planets : Jup1ter -planets : spectra 


\section{INTRODUCTION}

Water vapor is an important indicator of many physical processes occurring in Jupiter's atmosphere. In this paper we present an analysis of the global abundance, vertical distribution, and spatial variation of $\mathrm{H}_{2} \mathrm{O}$ in the 1 to 6 bar portion of Jupiter's troposphere. We used spectroscopic observations of Jupiter's $5 \mathrm{\mu m}$ atmospheric transmission window that were acquired from aircraft and spacecraft. Both datasets possess two special characteristics for studying $\mathrm{H}_{2} \mathrm{O}$ on Jupiter : there is no obscuration of Jovian $\mathrm{H}_{2} \mathrm{O}$ features by telluric $\mathrm{H}_{2} \mathrm{O}$ lines; and, Jovian $5 \mu \mathrm{m}$ flux is thermal emission which carries the spectral signature of $\mathrm{H}_{2} \mathrm{O}$ in the thermochemically important atmospheric level below the visible cloud tops. Our use of both datasets eliminates two major problems which have complicated previous interpretations of Jupiter's $5 \mathrm{\mu m}$ spectrum. First, the spectrum contains hundreds of absorption lines due to the vibration-rotation bands of at least seven gases. High spectral resolution is therefore required to separate Jovian $\mathrm{H}_{2} \mathrm{O}$ lines from other planetary absorptions. This is especially important when using both strong and weak lines to establish the vertical distribution of a non-uniformly mixed gas such as $\mathrm{H}_{2} 0$. At present, the 
highest resolution spectrum of Jupiter $\left(0.5 \mathrm{~cm}^{-1}\right)$ at $5 \mu \mathrm{m}$ without serious obscuration by telluric $\mathrm{H}_{2} \mathrm{O}$ was recorded from an aircraft. The second interpretive problem is a consequence of the large variation in $5 \mathrm{\mu m}$ cloud opacity between Jupiter's belts and zones. High spatial resolution is therefore required to relate the observed Jovian $\mathrm{H}_{2} \mathrm{O}$ mixing ratios to cloud morphology. At present, the $5 \mu \mathrm{m}$ spectrum of Jupiter with the highest spatial resolution (1.2 $2^{\circ}$ of Jovian latitude) was recorded from the Voyager spacecraft. This combination of observations therefore provides a unique database from which to model the abundance, vertical profile, and spatial variation of $\mathrm{H}_{2} \mathrm{O}$ in Jupiter's atmosphere. We find that the Jovian $0 / \mathrm{H}$ value is significantly depleted throughout the spectroscopically observable levels of Jupiter's atmosphere. We discuss ways in which this depleted $0 / \mathrm{H}$ value, combined with our recent determinations of enhanced $C / H$ and $N / H$ values from the same spectra (Bjoraker, Larson, and Kunde 1986, hereafter referred to as BLK), may constrain theories of Jupiter's origin. 


\section{OBSERVATIONS}

The observations of Jupiter at high spectral resolution were acquired with a Fourier spectrometer (Larson and Fink 1975) at the Kuiper Airborne Observatory (KAO) in 1975 Dec. The unapodized spectral resolution is $0.5 \mathrm{~cm}^{-1}$ in a spectral passband from 1800 to $2250 \mathrm{~cm}^{-1}$. The fleld of view covered the central 25" of Jupiter's 41.4" disk, from which we determined the average Jovian $\mathrm{H}_{2} \mathrm{O}$ abundance and vertical distribution between $-40^{\circ}$ and $40^{\circ}$ of Jovian latitude.

The observations at high spatial resolution were produced with the Infrared Interferometer Spectrometer (IRIS) on Voyager 1 during closest encounter in 1979 March (Hanel et al 1979, 1980). These data have the advantages of freedom from telluric absorption, absolute radiance calibration, and spatial resolution as high as $1.2^{\circ}$ of Jovian latitude at closest approach. The apodized spectral resolution is $4.3 \mathrm{~cm}^{-1}$ in a spectral passband from 180 to $2250 \mathrm{~cm}^{-1}$. We used the portion of this spectrum from 1800 and $2250 \mathrm{~cm}^{-1}$ to measure the spatial variation of $\mathrm{H}_{2} \mathrm{O}$ from the most transparent Hot Spots in the North Equatorial Belt to the cold, cloudy areas in the Equatorial and North Tropical Zones. IRIS acquired 25,000 planetary spectra, but 
the SNR at $5 \mu \mathrm{m}$ of an individual scan $1 \mathrm{~s}$ approximately 1 , so it is necessary to average a number of spectra for analysis. We selected ensembles of Voyager 1 IRIS $5 \mathrm{~mm}$ spectra to characterize the following regions on Jupiter : the Hot Spots in the North Equatorial Belt (NEB-Hot), South Equatorial Belt Hot Spots (SEB-Hot), the Equatorial Zone (EQZ), and an average of the regions on the planet which have the coldest $5 \mathrm{\mu m}$ brightness temperatures (Cold Zones). The Cold Zones ensemble includes spectra from the North Tropical Zone and the cloudiest portions of the Equatorial and South Temperate Zones. A fifth ensemble (Calib) has the same spatial resolution as the observations of Jupiter conducted from the KAO. This dataset was used to calibrate the $5 \mathrm{\mu m}$ absolute radiance of the airborne data. We used the following criteria to distinguish between Jupiter's belts and zones.

1. $5 \mu \mathrm{m}$ brightness temperature $\left(T_{5}\right)$. Ground-based maps of the Jovian disk at $5 \mu \mathrm{m}$ (Terrile 1978, Terrile et al 1979) show an enormous variation between belts and zones. Therefore, the brightness temperature of Jupiter at $5 \mathrm{\mu m}$ in the IRIS data was the primary criterion in selecting our spectral ensembles. We used the brightness temperature averaged over the 1950 to $2150 \mathrm{~cm}^{-1}$ portion of Jupiter's spectrum. Hot spots were defined as regions in which $\mathrm{T}_{5}$ was greater than $240 \mathrm{~K}$, while IRIS spectra with $\mathrm{T}_{5}<210 \mathrm{~K}$ were included in the Cold Zones ensemble. 
2. Latitudinal extent (LAT). The center point of the IRIS field of view was restricted to a swall range of latitudes for inclusion in the NEB-Hot, SEB-Hot, and EQZ ensembles, while the Cold Zones and Calib ensembles include spectra whose center point extends from $-41^{\circ}$ to $+41^{\circ}$ latitude. Spectra of all Jovian longitudes were included in each ensemble.

3. Emission angle $(\theta)$. The emission angle $\theta$ was chosen to be less than $30^{\circ}$ for the Hot Spots, while $\theta<34^{\circ}$ for the EQZ and $\theta<45^{\circ}$ for the Cold Zones ensemble to ensure inclusion of enough IRIS spectra to give an adequate SNR for each dataset.

4. Spatial resolution $(\Delta \lambda)$. The spatial resolution $\Delta \lambda$, expressed in degrees of projected Jovian latitude, was better than $7^{\circ}$ for the Hot Spots and $9.5^{\circ}$ for the zone ensembles. The Calib ensemble included spectra whose field of view on Jupiter encompassed the $-40^{\circ}$ to $+40^{\circ}$ latitude range for use in calibrating the alrborne observations.

5. $45 \mu \mathrm{m}$ brightness temperature $\left(\mathrm{T}_{45}\right)$. We applied another brightness temperature criterion to improve the homogeneity of the ensembles. The brightness temperature at $45 \mu \mathrm{m}$ is sensitive to the $\mathrm{NH}_{3}$ cloud opacity at 0.7 bars. This additional criterion helps to ensure that spectra of cloud-free and cloudy areas are not averaged together in our Hot Spot and zone ensembles.

The values of the above parameters for spectra in our five ensembles are summarized in Table 1. Some of the 
averaged characteristics of each ensemble are listed in Table 2. The size of the ensemble is larger for Jupiter's zones than for the Hot Spots in order to maintain an adequate SNR. The large value of the median spatial resolution for the calibration ensemble matches the field of view on Jupiter from the KAO. We defined a parameter $H$ to characterize the homogeneity of each ensenble. H is equal to the peak $5 \mu \mathrm{m}$ radiance of the ensemble divided by the component of the standard deviation of the radiance that is a real variation in Jupiter's spectrum, rather than instrumental noise. This variation is due to averaging together regions of Jupiter with differing amounts of cloud cover. This calculation is described in Bjoraker (1985). Inspection of Table 2 shows that the Equatorial Zone ensemble is quite heterogeneous (i. e. low $H$ ) and, therefore, a region of greatly varying cloud cover. The NEB Hot Spots, on the other hand, represent a fairly homogeneous sample. 
III. WATER VAPOR IN JUPITER'S ATMOSPHERE
A. OVERVIEW

Our procedure for interpreting Jupiter's infrared spectrum at $5 \mu \mathrm{m}$ consists of generating a synthetic spectrum from a radiative transfer model, comparing it to the observed airborne or IRIS data, and iterating parameters in the model atmosphere until the synthetic spectrum agrees with the observations within error limits. We used a spectrum synthesis program developed by Kunde and Maguire (1974). This algorithm computes the monochromatic absorption spectrum by numerically summing the contributions of many individual molecular absorption lines. The transmittance between each of 35 layers and the top of the atmosphere and the emergent radiance are calculated. The synthetic radiance is then convolved with the instrument function at a resolution of $0.5 \mathrm{~cm}^{-1}$ or $4.3 \mathrm{~cm}^{-1}$ for comparison with the airborne and Voyager data, respectively.

An important model parameter is the tropospheric temperature - pressure profile. The temperature at 1 bar is $165 \mathrm{~K}$ (Lindal et al 1981). We calculated the temperature between 1 and 7 bars assuming a dry adiabatic lapse rate 
with the hydrogen mole fraction, $\mathrm{qH}_{2}$, equal to 0.897 (Gautier et al 1981). The variation of the specific heat of $\mathrm{H}_{2}$ with temperature was taken into account.

The shapes of the absorption lines were described by collisionally-broadened Lorentz profiles. For all lines of $\mathrm{H}_{2} \mathrm{O}$ we used a value of $0.08 \mathrm{~cm}^{-1} / \mathrm{atm}$ for the weighted mean of the $\mathrm{H}_{2} \mathrm{O}-\mathrm{H}_{2}$ and $\mathrm{H}_{2} \mathrm{O}-\mathrm{He}$ broadening coefficients. The temperature dependence of the Lorentz half-width was assumed to be $\mathrm{T}^{-0.5}$, in accord with kinetic theory. Laboratory measurements of the $\mathrm{H}_{2}$ broadening coefficient as functions of frequency and temperature are needed to improve atmospheric models of Jupiter.

We included a massive absorbing cloud with a normal optical thickness of 2.93 at $5 \mu \mathrm{m}$ and a base at 2.1 bars, where $T=210 \mathrm{~K}$. The optical thickness was calculated by adding sufficient particle opacity to our gas-only model atmosphere until the calculated radiance at $2130 \mathrm{~cm}^{-1}$ matched the observed value in the airborne and IRIS Jupiter spectra. The cloud base temperature was inferred by matching observed and calculated values of the continuum radiance at the long and short wavelength ends of Jupiter's $5 \mu \mathrm{m}$ window. We describe this procedure in detail in Bjoraker, Kunde, and Larson (1986), hereafter referred to as BKL.

In Fig. 1 we compare the observed airborne spectrum of Jupiter to our "best-fit" synthet1c spectrum generated from our radiative transfer model. Absorption features at $5 \mu \mathrm{m}$ 
are due to the following gases in Juplter's atmosphere: $\mathrm{NH}_{3}$. $\mathrm{PH}_{3}, \mathrm{CH}_{4}, \mathrm{CH}_{3} \mathrm{D}, \mathrm{CO}, \mathrm{GeH}_{4}$, and $\mathrm{H}_{2} \mathrm{O}$. The abundances of all of these gases except $\mathrm{H}_{2} \mathrm{O}$ have been derived from the same a1rborne dataset and they were reported in a separate publication (BLK). The $\mathrm{H}_{2} \mathrm{O}$ absorption features visible in the airborne and Voyager IRIS Jupiter data belong to the $v_{2}$ fundamental vibration-rotation band centered at $1595 \mathrm{~cm}^{-1}$. The 1900 to $2150 \mathrm{~cm}^{-1}$ portion of Jupiter's spectrum is the best region for analyzing isolated $\mathrm{H}_{2} \mathrm{O}$ lines in this band. strong $\mathrm{NH}_{3}$ absorption dominates the 1800 to $1900 \mathrm{~cm}^{-1}$ end of the $5 \mu \mathrm{m}$ window and strong $\mathrm{PH}_{3}$ lines interfere with the few remaining weak $\mathrm{H}_{2} \mathrm{O}$ lines at frequencies higher than $2150 \mathrm{~cm}^{-1}$. Approximately $40 \mathrm{H}_{2} \mathrm{O}$ lines are evident in this interval at the $0.5 \mathrm{~cm}^{-1}$ resolution of the data. Vertical ines denote the positions of $21 \mathrm{H}_{2} \mathrm{O}$ absorption lines used in our analygis as well as the pressure levels where they are formed in Jupiter's atmosphere.

The bottom trace indicates the pressure level in our model where the gaseous optical depth equals unity as a function of wavenumber across the $5 \mu \mathrm{m}$ window. The maximum contribution to the thermal emission originates at the Indicated pressure level at each wavenumber. Pressure induced absorption by $\mathrm{H}_{2}$ determines the deepest level of Jupiter's troposphere that can be probed at $5 \mu \mathrm{m}$. Unit optical depth due to $\mathrm{H}_{2}$ takes place near 5 bars at $1900 \mathrm{~cm}^{-1}$. The most transparent part of Jupiter's $5 \mu \mathrm{m}$ window is at $2130 \mathrm{~cm}^{-1}$ where one can "see" as deep as 
7 bars. Most spectral features are formed in the 2 to 5 bar region. We therefore have a set of weighting functions which sample deep levels in Jupiter's atmosphere. In the next section we use $\mathrm{H}_{2} \mathrm{O}$ lines of varying strength to infer the vertical distribution of $\mathrm{H}_{2} \mathrm{O}$ between 2 and 6 bars in Jupiter's atmosphere. 


\section{B. THE VERTICAL DISTRIBUTION OF $\mathrm{H}_{2} \mathrm{O}$}

In this section we use the high spectral resolution airborne observations of Jupiter to derive the average abundance of $\mathrm{H}_{2} \mathrm{O}$ over the -40 to $+40^{\circ}$ latitude region of the planet. We examine several model distributions of $\mathrm{H}_{2} \mathrm{O}$ varying by a factor of 1000 in mole fraction. The presence of $\mathrm{H}_{2} \mathrm{O}$ lines of greatly varying strength across Jupiter's $5 \mu \mathrm{m}$ transmission window permits us to distinguish between these diverse models and it allows us to determine the vertical distribution of $\mathrm{H}_{2} \mathrm{O}$ in the 2 to 6 bar portion of Jupiter's troposphere.

We investigated four vertical distributions of $\mathrm{H}_{2} \mathrm{O}$ in Jupiter's troposphere by comparing model calculations to the airborne observations. The $\mathrm{H}_{2} \mathrm{O}$ mole fraction $\left(\mathrm{qH}_{2} \mathrm{O}\right)$ profiles as functions of temperature and pressure are displayed in Fig. 2 for the four candidate distributions. All profiles are constrained to follow the smaller value of either the saturated vapor pressure relation or the prescribed $\mathrm{H}_{2} \mathrm{O}$ partial pressure. Profile 1 is limited to a maximum value for $\mathrm{qH}_{2} \mathrm{O}=1 \times 10^{-6}$. Profile 2 is also height independent for $P>2$ bars but it has a larger value of $\mathrm{qH}_{2} \mathrm{O}=4 \times 10^{-6}$. Profile 3 also has a value of $\mathrm{qH}_{2} \mathrm{O}=4 \times 10^{-6}$ between 2 and 4 bars. However, $\mathrm{qH}_{2} \mathrm{O}$ increases with depth between 4 and 6 bars reaching a value of $3 \times 10^{-5}$ at 6 bars. 
Finally, we examined a limiting case (Profile 4) in which $O / H=8.34 \times 10^{-4}$ in Jupiter's deep atmosphere, the same value as in the photosphere of the Sun (Lambert 1978). The corresponding value of $\mathrm{qH}_{2} \mathrm{O}=1.5 \times 10^{-3}$. In chemical equilibrium models of Jupiter (Lewis 1969, Weidenschilling and Lewis 1973) condensation takes place at 5 bars to form a massive $\mathrm{H}_{2} \mathrm{O}$ cloud. The remaining gaseous $\mathrm{H}_{2} \mathrm{O}$ would closely follow the saturated vapor pressure curve for $P<5$ bars, as shown by Profile 4 in Fig. 2.

In Fig. 3 we illustrate how the large difference in $\mathrm{qH}_{2} \mathrm{O}$ between each of these models affects the spectrum of Jupiter at $5 \mu \mathrm{m}$. We calculated the absorption spectrum at $5 \mu \mathrm{m}$ for three of the four distributions of $\mathrm{H}_{2} \mathrm{O}$ shown in Fig. 2 : Profiles 1, 3, and 4. Profile 2 is omitted because it is very similar to Profile 3 on the scale of this figure. All absorption lines are due to $\mathrm{H}_{2} \mathrm{O}$ alone. Continuum opacity due to pressure induced absorption by $\mathrm{H}_{2}$ is included - this is responsible for the slope evident in the top spectrum. A simple absorbing cloud layer is also present in the model. The $5 \mu \mathrm{m}$ optical thickness of the cloud was adjusted to match the observed radiance in the transparent mini-window at $2130 \mathrm{~cm}^{-1}$ in the airborne Jupiter spectrum. The three spectra in Fig. 3 are therefore on the same absolute radiance scale. Absorption by the wings of $\mathrm{H}_{2} \mathrm{O}$ absorption lines is responsible for the dramatic difference in the continuum level between the three profiles. Thus, the continuum level as well as 1 ine to continuum ratios for 
strong and weak $\mathrm{H}_{2} \mathrm{O}$ lines provide important diagnostics to allow us to measure the Jovian $\mathrm{H}_{2} \mathrm{O}$ abundance from the airborne data.

We define three categories of $\mathrm{H}_{2} \mathrm{O}$ lines based on their measured strength, $\mathbf{S}$, in the laboratory at $296 \mathrm{~K}$.

(1) Strong lines : $\mathrm{S}>10^{-1} \mathrm{~cm}^{-1} / \mathrm{cm}$-amagat.

(2) Medium strength lines : $10^{-2}<\mathrm{S}<10^{-1} \mathrm{~cm}^{-1} / \mathrm{cm}$-amagat.

(3) Weak lines : $s<10^{-2} \mathrm{~cm}^{-1} / \mathrm{cm}$-amagat.

Examples of each are marked in Fig. 3. Because the center of the $v_{2}$ band of $\mathrm{H}_{2} \mathrm{O}$ is near $1600 \mathrm{~cm}^{-1}$ the general trend is for the lines to become weaker as $v$ increases from 1900 to $2150 \mathrm{~cm}^{-1}$. We examine in Fig. 3 the behavior of each class of line as $\mathrm{qH}_{2} \mathrm{O}$ is increased from $1 \times 10^{-6}$ (top trace) to $4 \times 10^{-6}$ (center trace) to $1.5 \times 10^{-3}$ (bottom trace). For the very weakest lines (v)2100 $\mathrm{cm}^{-1}$ ) the line contrast increases with increasing abundance. For medium strength $\mathrm{H}_{2} \mathrm{O}$ lines the contrast between line and continuum increases from the top to center trace, but it decreases for the bottom distribution. Finally, for the very strongest $\mathrm{H}_{2} \mathrm{O}$ lines the contrast between line center and continuun is greatest for Profile 1 and it decreases in Profiles 3 and 4 . We use the different spectral behavior of $\mathrm{H}_{2} \mathrm{O}$ lines belonging to each strength class to constrain the vertical distribution of $\mathrm{H}_{2} \mathrm{O}$ on Jupiter. 
In addition to using line to continuum ratios to measure $\mathrm{H}_{2} \mathrm{O}$ on Jupiter we use the characteristics of partially resolved $\mathrm{H}_{2} \mathrm{O}$ absorption lines and the absolute radiance of the continuum. Fig. 3 clearly shows substantial differences between the three $\mathrm{H}_{2} \mathrm{O}$ profiles in the degree of blending of line pairs near 1920, 1940, 1990, 2040, and $2090 \mathrm{~cm}^{-1}$. The importance of continuum absorption by the wings of $\mathrm{H}_{2} \mathrm{O}$ lines is also shown in Fig. 3. The radiance in "mini-wndows" at 1930, 1980, and $2060 \mathrm{~cm}^{-1}$ for Profile 4 is approximately half the value calculated for Profile 1 when both spectra are normalized at $2130 \mathrm{~cm}^{-1}$. Fig. 1 indicates that the continuum in many $5 \mu \mathrm{m}$ mini-windows is formed near 6 bars, while the line cores are formed at higher altitudes. Consequently, the large value of $\mathrm{qH}_{2} \mathrm{O}$ at 6 bars in Profile 4 strongly increases the absorption in the wings of the $\mathrm{H}_{2} \mathrm{O}$ lines at $5 \mu \mathrm{m}$. The continuum radiance alone, however, is difficult to interpret because of uncertainties in modelling cloud absorption at $5 \mu \mathrm{m}$. There are large spatial variations in Jupiter's $5 \mu \mathrm{m}$ flux due to varying cloud opacity (Terrile 1978). In addition, the frequency dependence of the absorbing cloud is not known, although one study suggests that it is constant across the $5 \mu \mathrm{m}$ window (Bezard et al 1983). In this study we will rely primarily on line to continuum ratios and line profiles to distinguish between different $\mathrm{H}_{2} \mathrm{O}$ distribuions for Jupiter.

We present in Figs. 4 - 6 a comparison of the observed Jupiter spectrum with spectra calculated using the four 
candidate $\mathrm{H}_{2} \mathrm{O}$ distributions. These figures show three smell portions of Jupiter's 5 um transmission window. CFor an

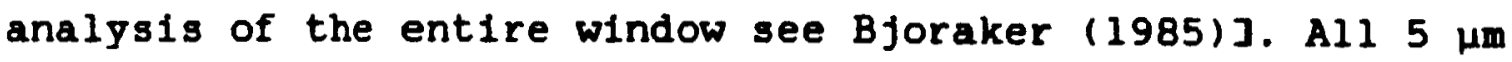
gaseous absorbers are included. For gases other than $\mathrm{H}_{2} \mathrm{O}$ we used the mole fractions previously reported by BLK. The frequency interval between 1962 and $2002 \mathrm{~cm}^{-1}$ in Fig. 4 includes seven $\mathrm{H}_{2} \mathrm{O}$ lines from all three strength classes. The top trace was calculated using Profile $1\left(\mathrm{gH}_{2} \mathrm{O}=1 \times 10^{-6}\right)$. The synthetic $\mathrm{H}_{2} \mathrm{O}$ lines are in all cases insufficiently absorbing. The disagreement is greatest for weak lines 3 and 6, but the mismatch is obvious for all but the very strongest line 5 . We reject Profile 1 from further consideration. A larger abundance of $\mathrm{H}_{2} \mathrm{O}$ for $\mathrm{P}>2$ bars is therefore required to fit the observations.

The bottom trace was calculated using $\mathrm{H}_{2} \mathrm{O}$ Profile 4 . The continuum level, normalized to the observations at $2130 \mathrm{~cm}^{-1}$, is in obvious disagreement with the observed data in this wavelength region. In addition, the wings of adjacent $\mathrm{H}_{2} \mathrm{O}$ lines are much broader in Profile 4 than in the Jupiter data. This is illustrated by 1 ines 1 and 2 as well as by lines 4, 5, and 6. Another diagnostic is the degree of blending between adjacent $\mathrm{H}_{2} \mathrm{O}$ lines. For example, lines 4 and 5 calculated from Profile 4 are blended to a much greater degree than in the observations. Finally, the effect of $\mathrm{H}_{2} \mathrm{O}$ wing absorption on absorption lines of other gases is significant. At $1972 \mathrm{~cm}^{-1}$ the $Q$-branch of the $2 v_{2}$ band of $\mathrm{PH}_{3}$ produces a prominent absorption. The $\mathrm{PH}_{3}$ mole fraction 
determined by $\mathrm{BLK}$ using $\mathrm{PH}_{3}$ features at $2100 \mathrm{~cm}^{-1}$ was used in each of the synthetic spectra shown in Fig. 4. Fig. I Indicates that the line formation region for the feature at $1972 \mathrm{~cm}^{-1}$ is the same as that for $\mathrm{PH}_{3}$ lines at $2100 \mathrm{~cm}^{-1}$. namely 5 bars. The only difference is that the $\mathrm{PH}_{3}$ feature in Fig. 4 is near the wing of line 2 , a strong $\mathrm{H}_{2} \mathrm{O}$ line. The effect of varying the $\mathrm{H}_{2} \mathrm{O}$ abundance at 5 bars from $10^{-6}$ to $10^{-3}$ is dramatic at $1972 \mathrm{~cm}^{-1}$. The $\mathrm{PH}_{3}$ line contrast is much smaller for Profile 4 than in our Jupiter spectrum. Profile 4 therefore provides a very poor $\mathrm{f} 1 \mathrm{t}$ to the $\mathrm{PH}_{3}$ and $\mathrm{H}_{2} \mathrm{O}$ features in the wavelength region of Fig. 4.

In contrast, Profile 3 provides an excellent fit to all spectral features in Fig. 4. Line 7 at $1999 \mathrm{~cm}^{-1}$ is matched perfectly, blended profiles agree, the continuum level is right, and the $\mathrm{PH}_{3}$ feature is fit reasonably well. We now examine how well this profile fits other portions of Jupiter's 5 rm window.

In Fig. 5 Jupiter's spectrum between 2063 and $2093 \mathrm{~cm}^{-1}$ is compared with synthetic spectra calculated using $\mathrm{H}_{2} \mathrm{O}$ Profiles 2, 3, and 4. We again reject Profile 4 for similar reasons as cited in the previous figure. The continuum level is too low and the calculated spectrum predicts blended features that are not observed. For example, $\mathrm{H}_{2} \mathrm{O}$ line 10 , which is adjacent to a $\mathrm{PH}_{3}$ line at $2075 \mathrm{~cm}^{-1}$, is clearly separated in the observations, but Profile 4 predicts a single blended feature. In addition, the low frequency wing of line 8 and the high frequency wing of line 14 calculated 
using Profile 4 are too strongly absorbing. These spectroscopic features indicate that Profile 4 has far too much $\mathrm{H}_{2} \mathrm{O}$ at the 5 bar level.

The wavelength region shown in Fig. 5 includes a number of $\mathrm{H}_{2} \mathrm{O}$ lines which are very sensitive to $\mathrm{qH}_{2} \mathrm{O}$ between 4 and 6 bars. We used them to distinguish between Profiles 2 and 3. Weak $\mathrm{H}_{2} \mathrm{O}$ lines 10,11 , and 13 are $\mathrm{f}$ it much better by the height dependent Profile 3 than by Profile 2. In addition. the blend of $\mathrm{H}_{2} \mathrm{O}$ lines 13 and 14 is matched significantly better by Profile 3. Absorption by $\mathrm{GeH}_{4}$ may be responsible for the slight mismatch in the position of line 13 between the model and observed data. Iines 9 and 14 offer additional supporting evidence for Profile 3 .

The final portion of the $5 \mathrm{\mu m}$ window that we have examined for $\mathrm{H}_{2} \mathrm{O}$ absorption is shown in FIg. 6. Although Profile 4 clearly failed to match the observations in Figs. 4 and 5 , it is useful to re-examine this distribution at the high frequency end of Jupiter's 5 um window for two reasons. First, the continuum radiance for Profile 4 was normalized to the observed Jupiter spectrum at $2130 \mathrm{~cm}^{-1}$ by adjusting the $5 \mu \mathrm{m}$ optical thickness of an absorbing cloud. One could argue that the continuum mismatch between Profile 4 and the observations at the wavelengths shown in Figs. 4 and 5 is due to uncertainties in the frequency dependence of the cloud absorption in the model. Consequently, it is important to examine the spectrum near the normalization frequency to separate the contributions of $\mathrm{H}_{2} \mathrm{O}$ and clouds to 
the total continuum opacity. Second, reference to F1g. I indicates that the weak 11 nes between 2120 and $2150 \mathrm{~cm}^{-1}$ are formed near 5 bars. Significantly, we learned from Fig. 3 that these lines grow monotonically with increasing $\mathrm{qH}_{2} \mathrm{O}$ over the range we are examining for Jupiter. Consequently, these lines are the best ones to test whether the $\mathrm{H}_{2} \mathrm{O}$ abundance at 5 bars is near $10^{-3}$, as in Profile 4 , or whether $\mathrm{qH}_{2} \mathrm{O}=10^{-5}$, as modelled in Profile 3 .

In Fig. $6 \mathrm{H}_{2} \mathrm{O}$ lines 15 to 21 belong to the weakest strength category. All of these lines appear too strongly absorbing in the bottom trace (Prof 1le 4). Line 16, in particular, is stronger than the adjacent $\mathrm{PH}_{3}$ line in Profile 4; whereas the observations show that the $\mathrm{H}_{2} \mathrm{O}$ line is slightly weaker than the $\mathrm{PH}_{3}$ line. Absorption by $\mathrm{H}_{2} \mathrm{O}$ lines 17 to 21 depresses the continuum in Profile 4 substantially below the measured value. Thus, the line contrast, line blend, and continuum level criteria are all met much better by Profile 3 than by 4 .

We summarize below the degree to which each of our $\mathrm{H}_{2} \mathrm{O}$ profiles is consistent with the observed spectrum of Jupiter .

(1) Profile 1 consistently generates medium and weak $\mathrm{H}_{2} \mathrm{O}$ lines that are too weak. This profile is considered incompatible with the observations because $\mathrm{qH}_{2} \mathrm{O}$ is too small for $P>2$ bars.

(2) Profiles 2 and 3 are similar, but Profile 3 provides demonstrably better fits to isolated weak $\mathrm{H}_{2} \mathrm{O}$ lines and to 
the profiles of blended $\mathrm{H}_{2} \mathrm{O}$ lines. Th1s 1mplies that the $\mathrm{H}_{2} \mathrm{O}$ abundance is larger at levels where weak lines are formed ( 5 to 6 bars) than where strong and medium strength lines form ( $P<4$ bars).

(3) Profile 4 produces too much absorption in the center of the weakest $\mathrm{H}_{2} \mathrm{O}$ lines. In particular, the weak $\mathrm{H}_{2} \mathrm{O}$ lines between 2120 and $2150 \mathrm{~cm}^{-1}$ constitute the most direct evidence that $\mathrm{H}_{2} \mathrm{O}$ is severely depleted at the 5 bar level. (4) Profile 4 adversely affects the line to continuum ratios for gases other than $\mathrm{H}_{2} \mathrm{O}$. The calculated wings of the $\mathrm{H}_{2} \mathrm{O}$ lines are so strongly absorbing that adjacent $\mathrm{PH}_{3}$ lines, for example, appear much weaker in the "solar" Profile 4 than in the observations. This problem is avoided with Profile 3 because $\mathrm{qH}_{2} \mathrm{O}$ is much less at 5 bars than in Profile 4. (5) Profile 4 does not simulate well the overall continuum level across Jupiter's $5 \mu \mathrm{m}$ spectrum. Profile 3 generates a much more satisfactory fit.

From these generalizations it appears that Profiles 1 and 4 fail to meet multiple criteria in matching the observations. Profile 2 is much better, but we were not able to match strong and weak $\mathrm{H}_{2} \mathrm{O}$ lines simultaneously using a constant value for $\mathrm{qH}_{2} \mathrm{O}$ at pressures greater than 2 bars. Prof 1 le 3 . on the other hand, closely reproduces all spectral features that we considered diagnostic of the abundance and distribution of Jovian $\mathrm{H}_{2} \mathrm{O}$. We therefore adopt Profile 3 for our inferred $\mathrm{H}_{2} \mathrm{O}$ vertical distribution in Jupiter's troposphere. It successfully reproduces the observed line 
to continuum ratios for twenty-one relatively unblended $\mathrm{H}_{2} \mathrm{O}$ absorption lines in our airborne data and its predicted continuum level is in good agreement with the observations. To summarize Profile 3 , the $\mathrm{H}_{2} \mathrm{O}$ mole fraction follows the saturated vapor law for $T<204 \mathrm{~K}$, (where $P(2$ bars), $\mathrm{qH}_{2} \mathrm{O}=4 \times 10^{-6}$ in the 2 to 4 bar range, and it increases to $3 \times 10^{-5}$ at 6 bars. The corresponding $\mathrm{H}_{2} \mathrm{O}$ scale height is $7.2 \mathrm{~km}$ for the 4 to 6 bar pressure range, or equivalently, between 254 and $287 \mathrm{~K}$.

We conclude that the $\mathrm{H}_{2} \mathrm{O}$ abundance on Jupiter is substantially below thermochemical predictions for the 2 to 6 bar region of the troposphere, at least for the central portion of the planet observed from the KAO. In the next section we examine the spatially resolved Voyager IRIS observations to determine if this conclusion applies to Juplter's zones and belts as well. 


\section{THE SPATIAL VARIATION OF $\mathrm{H}_{2} \mathrm{O}$}

In the preceding section we used the airborne observations at $5 \mathrm{\mu m}$ to characterize the average $\mathrm{H}_{2} \mathrm{O}$ abundance for the region between $-40^{\circ}$ and $+40^{\circ}$ latitude on Jupiter. However, Jupiter exhibits enormous variations in brightness temperatures on spatial scales of only a few degrees of latitude. High spatial resolution spectroscopic measurements are therefore needed to determine if the gas composition also varies between Jupiter's belts and zones. In this section we use the Voyager IRIS observations at $5 \mu \mathrm{m}$ to measure the spatial variation of $\mathrm{H}_{2} \mathrm{O}$ between several diverse regions of Jupiter's atmosphere. These range from the most transparent Hot Spots in the North Equatorial Belt to the cloud-covered zones with the coldest $5 \mu \mathrm{m}$ brightness temperatures. Zones are believed to be regions of upward motion in which parcels of the atmosphere become saturated in $\mathrm{H}_{2} \mathrm{O}$ and form water ice clouds. Belts, on the other hand, are thought to be regions of downward motion in which atmospheric parcels, depleted in $\mathrm{H}_{2} \mathrm{O}$ vapor, are transported to deeper levels to complete the cycle. This dynamical argument predicts that more $\mathrm{H}_{2} \mathrm{O}$ should be present in zones than in belts. Thus, measurements of the $\mathrm{H}_{2} \mathrm{O}$ abundance in $5 \mu \mathrm{m}$ spectra of zones should allow us to 
discriminate between two very different interpretations of the distribution of $\mathrm{H}_{2} \mathrm{O}$ on Jupiter that have been previously mentioned, but never critically analyzed.

One idea is that zones represent a hidden reservoir of $\mathrm{H}_{2} \mathrm{O}$ in which the $\mathrm{O} / \mathrm{H}$ ratio is verg nearly equal to that in the solar photosphere. This model attempts to reconcile central disk measurements of $\mathrm{H}_{2} \mathrm{O}$, which indicate a substantial depletion, with expectations that the deep atmosphere of Jupiter has a solar $0 / \mathrm{H}$ ratio. Current theories for the formation of the glant planets predict $\mathrm{qH}_{2} \mathrm{O}>10^{-3}$. We have just shown with the KAO data, however, that the central disk value of $\mathrm{qH}_{2} \mathrm{O}$ is no more than $4 \times 10^{-6}$ in the 2 to 4 bar region of Jupiter's atmosphere. In the "hidden reservolr" scenario, this discrepancy in $\mathrm{H}_{2} \mathrm{O}$ abundance is explained in the following way. Jupiter's zones contribute very little $5 \mu \mathrm{m}$ flux to our alrborne spectrum, for example, because of cold, optically thick clouds. Central disk observations would therefore contain information only about the $\mathrm{H}_{2} \mathrm{O}$ abundance in the belts and Hot Spots where all of the $5 \mu \mathrm{m}$ flux originates. According to this model, belts are extremely desiccated with respect to the adjacent zones. Condensation efficiently removes nearly all of the $\mathrm{H}_{2} \mathrm{O}$ from the gas phase, and subsequent downward motion in the belts returns very dry "alr" to the deep troposphere. Since only this dry air is observed, a measured depletion of $\mathrm{qH}_{2} \mathrm{O}$ is still consistent with an overall solar composition. 
As an alternative to the "hidden reservoir" model. the observable levels of Jupiter's atmosphere may simply be depleted in oxygen by a factor of the order of 100. In this model atmospheric dynamics is less important in characterizing the spatial variation of $\mathrm{H}_{2} \mathrm{O}$ since belts and zones would have roughly comparable, but substantially depleted, amounts of $\mathrm{H}_{2} \mathrm{O}$ at lower tropospheric levels. Clearly, it is important to measure $\mathrm{qH}_{2} \mathrm{O}$ at the same pressure level in both belts and zones to distinguish between these two hypotheses.

In Figs. 7 and 8 we show the four IRIS ensembles characterizing both belts and zones on Jupiter. The diversity of these regions is shown by the radiance scales. The peak radiance is a factor of 20 higher in the NEB-Hot ensemble in Fig. 7 than in the Cold Zone average in Fig. 8. With such an enormous variation in radiance between belt and zone spectra, one might expect substantial differences in the appearance of their 5 m spectra. However, when each spectrum is plotted on a scale normalized to its peak radiance, they appear nearly identical. Note especially the similarities between the NEB-Hot ensemble in Fig. 7 and the EQZ ensemble in Fig. 8. Common features include transmission peaks at $1930,1980,2080$, and $2130 \mathrm{~cm}^{-1}$ and approximately 30 absorption lines. The positions of fourteen prominent $\mathrm{H}_{2} \mathrm{O}$ features are marked by arrows in Figs. 7 and 8 . These are blends of $\mathrm{H}_{2} \mathrm{O}$ lines with other features that are seen at higher spectral resolution in the airborne data. Only those 
features in which $50 \%$ or more of the absorption is due to $\mathrm{H}_{2} \mathrm{O}$ were chosen for analysis. Strong $\mathrm{H}_{2} \mathrm{O}$ lines in the IRIS data are formed between 2 and 3 bars in Jupiter's atmosphere, whlle weak lines are formed between 4 and 5 bars. Consequently, the IRIS data allow us to distinguish between height dependent and constant $\mathrm{H}_{2} \mathrm{O}$ profiles for Jupiter's belts and zones.

Accompanying each observed spectrum in Figs. 7 and 8 is a "best-fit" synthetic spectrum generated with our radiative transfer model. The cloud structure and gas composition, except $\mathrm{H}_{2} \mathrm{O}$, were fixed using the baseline model described in Section IIIA. The "best-fit" $\mathrm{H}_{2} \mathrm{O}$ profiles to the IRIS spectra are displayed in Fig. 9. Only two height dependent profiles were needed to characterize the distribution of $\mathrm{H}_{2} \mathrm{O}$ in our four ensembles. One (Profile NEB) applies just to the NEB-Hot ensemble; the other (Profile ZONE) applies equally to the SEB-Hot and the two zonal averages. Both profiles are compared in Fig. 9 with two extreme distributions : solar $0 / \mathrm{H}\left(\mathrm{qH}_{2} \mathrm{O}=1.5 \times 10^{-3}\right) \bmod 1 \mathrm{fled}$ by vapor pressure saturation for $\mathrm{T}<273 \mathrm{~K}$ (Profile 4); and, globally depleted $0 / \mathrm{H}\left(\mathrm{qH}_{2} \mathrm{O}=\right.$ $1 \times 10^{-6}$. Profile 1). Recall that nefther extreme distribution matched the airborne observations of Jupiter.

The similarity of our best-fit $\mathrm{H}_{2} \mathrm{O}$ profiles for the SEB-Hot Spots and the two zonal averages is very surprising because these regions are thought to be quite different dynamically. These spatial averages differ substantially in radiance, but the line-to-continuum ratios for the $\mathrm{H}_{2} \mathrm{O}$ 
features in each ensenble are nearly the same. This behavior demonstrates that the abundance of $\mathrm{H}_{2} \mathrm{O}$ in Juplter's atmosphere is not a strong function of spatial location. Profile ZONE should then closely approximate the distribution inferred from central disk observations as well. This expectation is confirmed by our analysis of the KAO data. Profile 3 in Fig. 2, the best-fit central disk distribution, is comparable to Profile ZONE in Fig. 9, differing only by a factor of 2 in the 2 to 4 bar region. Only the NEB Hot spots have an $\mathrm{H}_{2} \mathrm{O}$ abundance that differs from the global average, but only by small factors in the P<4 bar region, as discussed below.

The different $\mathrm{H}_{2} \mathrm{O}$ distribution in Jupiter's North Equatorial Belt is illustrated with the superposition of the two IRIS Hot Spot averages in Fig. 10. The spectra are normalized such that their continua match in the 2000 to $2150 \mathrm{~cm}^{-1}$ region. This procedure permits a direct comparison of the line to continuum ratios of the twelve $\mathrm{H}_{2} \mathrm{O}$ absorption features indicated by arrows in Fig $=10$. All of the $\mathrm{H}_{2} \mathrm{O}$ features are stronger in the SEB than in the NEB Hot Spots, but the discrepancy in line strengths is greatest for the absorption lines for $v<2050 \mathrm{~cm}^{-1}$. This implies that $\mathrm{qH}_{2} \mathrm{O}$ for $\mathrm{P}<4$ bars is significantly less in the NEB than elsewhere on Jupiter. The integrated column abundance of $\mathrm{H}_{2} \mathrm{O}$ above the 3 bar level, for example, is $11 \mathrm{~cm}$-am in the SEB Hot Spots but only $1.2 \mathrm{~cm}$-am in the NEB Hot Spots. However, the $\mathrm{H}_{2} \mathrm{O}$ lines at 2066 and $2090 \mathrm{~cm}^{-1}$ are 
of comparable strength in the NEB and SEB Hot Spots. These lines are formed at deeper levels in Jupiter's atmosphere (between 4 and 5 bars), so the $\mathrm{H}_{2} \mathrm{O}$ profile for $\mathrm{P}>4$ bars is very similar for both regions. Thus, the integrated $\mathrm{H}_{2} \mathrm{O}$ column abundances differ by only a factor of 2 at 4.3 bars, and the values are essentially the same at 6 bars.

We therefore interpret the IRIS belt and zone spectra in the following way. The line forming region for each spectrum is in Jupiter's deep troposphere below the massive absorbing cloud at the 2 bar level [see BKL for a detalled analysis of Jupiter's cloud structure using IRIS $5 \mu \mathrm{m}$ spectra]. This cloud acts as a neutral density filter. relatively transparent in the belts and highly absorbing in the zones. The strengths of the $\mathrm{H}_{2} \mathrm{O}$ features in the belt and zone spectra therefore indicate the $\mathrm{H}_{2} \mathrm{O}$ abundance at the same pressure levels in both types of regions. Since Profile ZONE fits belts and zones we therefore conclude that the abundance and distribution of $\mathrm{H}_{2} \mathrm{O}$ in Jupiter's troposphere is nearly independent of the overlying cloud cover that distinguishes these regions morphologically. The only spatial variation in Jupiter's $\mathrm{H}_{2} \mathrm{O}$ abundance is a depletion factor of 3 for the 2-4 bar level of the NEB Hot Spots with respect to the rest of the planet.

Our analysis extends two previous studies of $\mathrm{H}_{2} \mathrm{O}$ on Jupiter using the IRIS $5 \mu \mathrm{m}$ spectra. Kunde et al (1982) analyzed $5 \mu \mathrm{m}$ IRIS observations of the NEB Hot Spots using the same average of 51 spectra that comprised our NEB 
ensemble. Their model included an opaque lower boundary at $\mathrm{T}=279 \mathrm{~K}$ and a uniformly mixed gray haze at higher altitudes. They inferred a distribution in which $\mathrm{qH}_{2} \mathrm{O}$ increased from $1 \times 10^{-6}$ at 2.5 bars to $3 \times 10^{-5}$ at 4 bars. Our best-fit $\mathrm{H}_{2} \mathrm{O}$ profile NEB is similar to that of Kunde et al except for the pressure level at which $\mathrm{qH}_{2} \mathrm{O}$ increases. Th1s level is model dependent because of different boundary conditions and a different distribution of cloud opacity. Drossart and Encrenaz (1982) examined three IRIS ensembles to infer the average $\mathrm{H}_{2} \mathrm{O}$ abundance between $-30^{\circ}$ and $+30^{\circ}$ latitude on Jupiter as well as for areas in the North and South Equatorial Belts. Although their average spectrum included contributions from Jupiter's zones, Drossart and Encrenaz did not analyze regions with $5 \mu \mathrm{m}$ brightness temperatures less than $210 \mathrm{~K}$. They inferred values for $\left[\mathrm{H}_{2} \mathrm{O}\right] /\left[\mathrm{H}_{2}\right]$ of $4 \times 10^{-6}$ for their central disk average and for the NEB Hot Spots, while their value for the SEB Hot Spots was $7.2 \times 10^{-6}$. Drossart and Encrenaz adopted a height independent profile for $\mathrm{H}_{2} \mathrm{O}$ in Jupiter's atmosphere. They concluded that uncertainties in $\mathrm{H}_{2} \mathrm{O}$ linewidths did not allow them to distinguish between uniformly mixed profiles and ones in which there is a larger $\mathrm{H}_{2} \mathrm{O}$ abundance at great depth in Jupiter's atmosphere. 
D. DISCUSSION

In this study we have derived three vertical profiles for $\mathrm{H}_{2} \mathrm{O}$ in Jupiter's troposphere using a combination of central disk and spatially resolved observations of Jupiter's 5 mm spectrum. Profile 3 in Fig. 2 (KAO data analysis) provides the best fit to the central portion of Jupiter between -40 and $40^{\circ}$ latitude. The NEB profile in Fig. 9 matches the IRIS observations of Jupiter's Hot Spots in the North Equatorial Belt. The ZONE profile fits both the cloudy zones of Jupiter as well as the Hot spots in the South Equatorial Belt. The following conclusions result from comparing these three distributions.

1. The three $\mathrm{H}_{2} \mathrm{O}$ profiles are nearly identical in spite of the very different spatial coverage and spectral resolution to which they apply.

11. All best-fit distributions show height dependent behavior.

11. All best-fit distributions deviate significantly from the solar profile at all atmospheric levels observed at $5 \mu \mathrm{m}$ ( $P<6$ bars).

1v. The best-fit distributions do reveal spatial differences in Jupiter's $\mathrm{H}_{2} \mathrm{O}$ abundance, but only by small 
factors of about 3 in the NEB Hot spots above the $P=4$ bar level.

The implications of these results are discussed below.

1. Abundance and distribution of $\mathrm{H}_{2}$. We summarize the results of our $\mathrm{H}_{2}$ O analysis in Fig. 11. The best-fit profile to the KAO data (profile 3 in Fig. 2) is presented in

F1g. Il to show Its dependence on $\mathrm{H}_{2} \mathrm{O}$ Iines of different strength. We divided the $21 \mathrm{H}_{2} \mathrm{O}$ absorption lines used in our analysis into three categories : strong lines, formed between 2 and 3 bars; medium lines, formed near 4 bars; and, weak 11nes, formed near 6 bars. We extended the range of this profile by adding a fourth point, the global abundance of $\mathrm{H}_{2} \mathrm{O}$ on Jupiter from airborne observations at $2.7 \mathrm{\mu m}$. Larson et al (1984) reported an upper limit to $\mathrm{qH}_{2} \mathrm{O}$ of $3 \times 10^{-9}$ in the 0.7 to 1.2 bar pressure range.

We also display in Fig. 11 our best-fit $\mathrm{H}_{2} \mathrm{O}$ profile to Jupiter's SEB Hot Spots and its zones (Profile ZONE in Fig. 9) and Profile NEB, which fits the IRIS spectra of the NEB Hot spots. The ZONE profile is slightly drier (by about a factor of 2) than our KAO result in the 2 to 4 bar region. The NEB Hot Spots appear to be desiccated by a factor of 4 at $\mathrm{P}=3$ bars with respect to the rest of the planet. This is consistent with a dynamical mechanism that "dries out" regions that have significantly smaller cloud opacity at $5 \mu \mathrm{m}$. Note, however, that for P>4 bars the NEB profile is identical to both the KAO and ZONE distributions. Thus, we 
emphasize here as one of our principal results that separate analyses of Independent datasets differing substantially in their spectral and spatial resolution have led to comparable helght dependent distributions of $\mathrm{H}_{2} \mathrm{O}$ in Jupiter's atmosphere.

In contrast to this result, the thermochemical equilibrium model of Weidenschilling and Lewis predicts a substantially higher $\mathrm{H}_{2} \mathrm{O}$ abundance throughout the

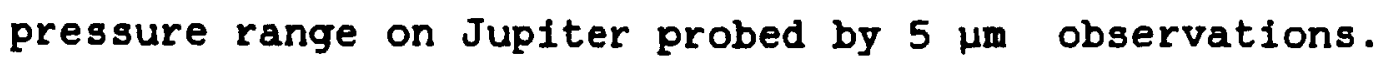
This model, Indicated as Profile 4 in Figs. 2 and 9, 18 shown by a dashed line in Fig. 11. At the 5 bar level where $T=273 \mathrm{~K}$, the saturated mole fraction of $\mathrm{H}_{2} \mathrm{O}$ equals the predicted value of $\mathrm{qH}_{2} \mathrm{O}$ for a solar composition atmosphere. This is where Weidenschilling and Lewis estimated that a massive water ice cloud would form in Jupiter's atmosphere. However, our observations show that $\mathrm{qH}_{2} \mathrm{O}$ at the 5 bar level is no higher than $10^{-5}$, a value that is undersaturated by a factor of 100 . We therefore exclude the presence of a massive water ice cloud at the 5 bar level in Jupiter's atmosphere.

Each of our derived $\mathrm{H}_{2} \mathrm{O}$ profiles exhibits height dependent behavior at $P<2$ bars as well as between 4 and 6 bars. Condensation readily explains the falloff for P<2 bars, but there 1s no obvious mechanism for changing the $\mathrm{H}_{2} \mathrm{O}$ mole fraction between 4 and 6 bars in Jupiter's atmosphere. At these deep levels some as yet unidentified chemical processes may be required to explain the 
observations. Photochemistry is not operative in th1s region because ultraviolet radiation does not penetrate this deep into the Jovian atmosphere. In order for chemical reactions to reduce $\mathrm{qH}_{2} \mathrm{O}$ from $3 \times 10^{-5}$ at 6 bars to $4 \times 10^{-6}$ near the 4 bar level, the proposed chemical reactants presumably would have to be at least as abundant as $\mathrm{H}_{2} \mathrm{O}$. However, there are very few gases in Jupiter's atmosphere with abundances high enough to deplete its $\mathrm{H}_{2} \mathrm{O}$ abundance noticeably by chemical reactions. One possible reaction might involve $\mathrm{NH}_{3}$ and $\mathrm{H}_{2} \mathrm{O}$. Lewis (1969) showed a phase dlagram for aqueous ammonia solutions in the atmospheres of the outer planets. However, our inferred $\mathrm{H}_{2} \mathrm{O}$ and $\mathrm{NH}_{3}$ mole fractions plot in the $\mathrm{H}_{2} \mathrm{O}$ ice stability field of Lewis' phase diagram, so phase changes involving $\mathrm{H}_{2} \mathrm{O}$ on Jupiter would result in the condensation of pure $\mathrm{H}_{2} \mathrm{O}$ ice rather than an aqueous ammonia solution. Thus, aqueous ammonia clouds are not expected to be present in Jupiter's atmosphere; therefore this mechanism is probably not responsible for Jupiter's observed $\mathrm{H}_{2} \mathrm{O}$ profile. Dynamics may be partially responsible for the observed sub-saturated behavior of $\mathrm{H}_{2} \mathrm{O}$ at $\mathrm{P}<6$ bars. However, we have fust shown that both belts and zones have similar vertical $\mathrm{H}_{2} \mathrm{O}$ distributions between 4 and 6 bars despite their meteorological differences. In situ measurements conducted from the Galileo entry probe may ultimately resolve some of these questions. 
2. Location of the $\mathrm{H}_{2}$ O cloud. We demonstrated above that the undersaturated values of $\mathrm{qH}_{2} \mathrm{O}$ near the 5 bar level preclude formation of the massive water ice cloud predicted in the solar composition model. In addition, a study of IRIS 5 um spectra (BKL) reports that there is no evidence for thermal emission from an optically thick $\mathrm{H}_{2} \mathrm{O}$ cloud in the 250 to $300 \mathrm{~K}$ portion of Jupiter's atmosphere, or equivalently, between 4 and 7 bars. A massive $\mathrm{H}_{2} \mathrm{O}$ cloud at 5 bars on Jupiter 13 therefore incompatible with the observed $5 \mu \mathrm{m}$ spectrum, unless the Jovian temperature prof lle is vastly different from that used in our model. Our baseline radiative transfer model and the calculated saturation vapor pressure curve (shown in Fig. 11) are based on the assumption that Jupiter's temperature profile is adiabatic between 1 and 5 bars. We have also investigated several different temperature profiles for $P>1$ bar. We find that Jupiter's temperature profile would have to be much colder and the lapse rate greatly subadiabatic in order for our measured $\mathrm{H}_{2} \mathrm{O}$ abundance to be close to saturation at 5 bars. Such a profile is inconsistent with Jupiter's observed heat flux, which requires a temperature profile very close to adiabatic to transport heat from the interior. We use our inferred height dependent $\mathrm{H}_{2} \mathrm{O}$ distribution to predict where the water ice cloud should be located on Jupiter. Fig. 11 shows that our derived $\mathrm{H}_{2} \mathrm{O}$ abundance curve intersects the saturated vapor pressure curve near $P=2$ bars. These gas phase $\mathrm{H}_{2} \mathrm{O}$ measurements imply that condensation of 
water 1ce would take place near the 2 bar level, where $\mathrm{T}=204 \mathrm{~K}$. We indicate the presence of our predicted water ice cloud in the portion of Fig. 11 marked "Inferred Cloud Structure". The presence of some kind of cloud layer near 2 bars is supported by modelling of the continuum radiance at the long and short wavelength ends of Juplter's $5 \mathrm{\mu m}$ window (see BKL). Thermal emission from optically thick clouds has a prominent temperature-sensitive signature at $5 \mathrm{\mu m}$. Our measurements also permit an estimate of the mass of Jupiter's $\mathrm{H}_{2} \mathrm{O}$ cloud. Our value for $\mathrm{qH}_{2} \mathrm{O}$ of $4 \times 10^{-6}$ at the saturation level is a factor of 400 smaller than that proposed in solar composition models. Consequently, our inferred $\mathrm{H}_{2} \mathrm{O}$ ice cloud is significantly less massive than the cloud proposed for the 5 bar level by Weidenschilling and Lewis.

3. Cloud composition. Our estimate of the location of Jupiter's $\mathrm{H}_{2} \mathrm{O}$ cloud $(\mathrm{P}=2$ bars $)$ is, colncidentally, the same level where $\mathrm{NH}_{3}$ and $\mathrm{H}_{2} \mathrm{~S}$ are expected to react to form an $\mathrm{NH}_{4} \mathrm{SH}$ cloud. This level is calculated using the value for $\mathrm{qNH}_{3}$ reported by BLK and using our atmospheric model (Larson et al 1984). The composition of the cloud may therefore include both $\mathrm{H}_{2} \mathrm{O}$ ice and $\mathrm{NH}_{4} \mathrm{SH}$, as shown schematically in Fig. 11. Bezard et al (1983) calculated the mass per unit area and the corresponding $5 \mathrm{\mu m}$ optical thickness for $\mathrm{H}_{2} \mathrm{O}$ ice clouds in equilibrium with $\mathrm{H}_{2} \mathrm{O}$ vapor for values of the Jovian $0 / H$ ratio ranging from solar to $10^{-3}$ times solar. They found that $\mathrm{H}_{2} \mathrm{O}$ ice can only marginally explain the 
observed spread in $5 \mu \mathrm{m}$ brightness temperatures between Juplter's belts and zones. Bezard et al concluded that an $\mathrm{NH}_{4} \mathrm{SH}$ cloud can more easily provide the required $5 \mathrm{~mm}$ opacity than is possible using $\mathrm{H}_{2} \mathrm{O}$ ice alone.

4. Global $\mathrm{O} / \mathrm{H}$ value. The value of $\mathrm{qH}_{2} \mathrm{O}$ between 2 and 4 bars on Jupiter $1 \mathrm{~s}$ no larger than $4 \times 10^{-6}$ in any of our $\mathrm{H}_{2} \mathrm{O}$ distributions. This value is about 100 times less than the saturated value of $\mathrm{qH}_{2} \mathrm{O}$ predicted by the solar composition model. The depletion factor decreases to about 50 at 6 bars, the deepest atmospheric level to which we can probe at $5 \mu \mathrm{m}$. We emphasize that the $\mathrm{H}_{2} \mathrm{O}$ profiles in Fig. 11 for Jupiter's belts and zones for P>4 bars are identical and they are depleted with respect to the "solar" model. This strongly suggests that there are no preferred regions on Jupiter with vastly different $\mathrm{H}_{2} \mathrm{O}$ abundances. Thus, the "hidden reservolr" hypothesis, in which the $0 / H$ ratio in Jupiter's cloud-covered zones is equal to the solar value, is inconsistent with the $5 \mu \mathrm{m}$ spectra. This result supports a global depletion of $0 / H$ in Jupiter's interior, but. alternatively, the increase in $\mathrm{qH}_{2} \mathrm{O}$ that we inferred for pressures between 4 and 6 bars may continue to deeper levels. In this case Jupiter may still retain a global $0 / \mathrm{H}$ ratio similar to that of the Sun. However, if the inferred value of $\mathrm{qH}_{2} \mathrm{O}$ at 6 bars is representative of the deep atmosphere, then Jupiter is globally depleted in oxygen by a factor of 50. This has significant consequences for models of Jupiter's origin. Other key elemental abundances 
(e.g. $\mathrm{C} / \mathrm{H}, \mathrm{N} / \mathrm{H}$ ) in Jupiter's atmosphere also differ from solar values. In section IV we review ways in which these anomalies could relate to Jupiter's origin and evolution. The Jovian $O / H$ ratio also affects the stability of the observed disequilibrium species, including $\mathrm{PH}_{3}, \mathrm{CO}$, and possibly $\mathrm{GeH}_{4}$. A depletion of oxygen would increase the stability of $\mathrm{PH}_{3}$ and $\mathrm{GeH}_{4}$, but it would make $\mathrm{CO}$ less stable. Further studies of the kinetics of chemical reactions under Jovian conditions is required to understand the conversion of carbon, phosphorus, and germanium between their reduced and oxidized forms. 
IV. COMPOSITIONAL CONSTRAINTS TO THE ORIGIN OF JUPITER

Measurements of the abundance of $\mathrm{CH}_{4}, \mathrm{NH}_{3}$, and $\mathrm{H}_{2} \mathrm{O}$ in Jupiter's atmosphere provide useful constraints in models describing the interiors of the glant planets. They also help to provide quantitative tests of various theories of planet formation. In this section we discuss some of the implications of our conclusions that Jupiter's atmosphere is globally enhanced in carbon and nitrogen, but perhaps depleted in oxygen with respect to the solar photosphere. A full theoretical analysis of the ways in which elemental abundances constrain models of Jupiter's origin is beyond the scope of this paper. Here we simply cite some recent models and we compare our inferred gas composition to model predictions in the few instances where quantitative estimates have been made.

In this paper and in BLK we have presented observational evidence for the enhancement of the global $\mathrm{C} / \mathrm{H}$ ratio on Jupiter by a factor of $3.6 \pm 1.2$, an enhancement of $N / H$ by $1.5 \pm 0.2$, and the depletion of $0 / H$ by perhaps a factor of 50 . We now exanine some recent models of Jupiter's internal structure to see if the spectroscopic evidence for global enrichment in carbon and nitrogen is Independently supported. Hubbard and Horedt (1983) used 
recent improvements in measurements of Jupiter's gravito field, helium mass fraction, and temperature profile to develop new models of Jupiter's interior. Their model consists of an inner "rock" core of iron and magnesium silicates, an outer "ice" core of $\mathrm{H}_{2} \mathrm{O}, \mathrm{CH}_{4}$, and $\mathrm{NH}_{3}$, and an outer hydrogen rich envelope. They derived a pressuredensity relation for Jupiter which is inconsistent with a solar composition envelope. The hellum mass fraction must be either larger than derived by Gautier et al (1981), or, more $11 \mathrm{kely}, \mathrm{CH}_{4}$ and $\mathrm{NH}_{3}$ must be enhanced by a factor of about 6 . The actual value depends on the equation of state in the $10^{5}$ to $5 \times 10^{6}$ bar pressure range in Jupiter's interior. Their model is sensitive to the Jovian gas composition only in this pressure range, but throughout the convective region the atmosphere is expected to be uniformly mixed.

We now examine two theoretical frameworks that have been proposed to explain the origin of the giant planets to see how measurements of the abundances of $C, N$, and 0 in Jupiter's atmosphere may help to distinguish between various models. Cameron (1978) proposed that the solar nebula fragmented into numerous giant gaseous protoplanets. De Campli and Cameron (1979) and Bodenheimer et al (1980) investigated the structure and evolution of such gaseous protoplanets. These objects are assumed to be chemically homogeneous condensations of solar composition gas and dust present in the primordial solar nebula. A 
small core forms later through sedimentation of grains toward the center of the planet.

An alternative scenario is provided in the nucleation model developed by Perri and Cameron (1974) and Mizuno (1980). In this model grains in the solar nebula accrete to form an initial core. When the mass of the core reaches a critical value the surrounding gaseous envelope undergoes hydrodynamic collapse onto the core. Internal heating could cause some of the ices to erode from the core, and subsequent convection would redistribute this material to enrich the outer atmospheric envelope.

An enrichment of carbon and nitrogen in the atmosphere of Jupiter is difficult to reconcile with either of the two theories of planet formation outlined above. Gautier and Owen $(1983,1985)$ pointed out that the homogeneous collapse, or giant gaseous protoplanet scenario, is in disagreement with available compositional data because it predicts a solar composition atmosphere. The nucleation, or accretion model, has independent observational support from studies of Jupiter's gravity harmonics, $\mathrm{J}_{2}$ and $\mathrm{J}_{4}$. Jupiter is believed to have a core of about 15 to 30 Earth masses (Hubbard and Horedt 1983). The homogeneous collapse model may not produce a core at all, or at least, the resulting core is smaller than that required by available gravity data, while the accretion model predicts a massive core for all of the outer planets. 
Gautier and Owen (1983, 1985) argued that the enhanced $\mathrm{C} / \mathrm{H}$ ratio in Jupiter's atmosphere provides strong compositional evidence for the accretion model. The accretion model may be correct, but three conditions must be satisfied in order for it to explain the observed enrichment of carbon and nitrogen in Jupiter's atmosphere. First, the core of proto-Jupiter had to contain not only silicates and water ice, but ammonia and methane as well, perhaps in the form of a clathrate. Second, after hydrodynamic collapse a significant amount of redistribution of core material had to take place in order to enrich the atmospheric envelope. Finally, this enrichment process had to enhance preferentially the abundance of $\mathrm{CH}_{4}$ and $\mathrm{NH}_{3}$ with respect to $\mathrm{H}_{2} \mathrm{O}$. However, lack of knowledge of the oxygen abundance at pressures greater than 6 bars on Jupiter makes this third restriction much less stringent than the first two.

The composition of Jupiter's core is not known. Refractory material certainly condensed to form a rocky inner core, but the composition of the outer core is model dependent. An important parameter is the temperature of the solar nebula at Jupiter's distance from the Sun. According to models of the temperature gradient in the solar nebula (Lewis 1974), water ice very likely condensed, but it is not certain that more volatile ices condensed at $5 \mathrm{AU}$. Lewis noted that a comparison of the regular satellite systems of Jupiter and Saturn may 
provide an estimate of the temperature fleld near these planets at the time of their formation. Ganymede and Callisto have incorporated water 1ce, but Titan was able to include $\mathrm{CH}_{4}$ and $\mathrm{NH}_{3}$ ices or clathrates as well due to its formation at a lower temperature. This may explain why Titan has an atmosphere containing large amounts of nitrogen and carbon (in the form of $\mathrm{N}_{2}$ and $\mathrm{CH}_{4}$ ) whereas Ganymede and Callisto do not have atmospheres at all, despite being about the same size as Titan. This satelite system comparison might constrain the composition of Jupiter's core because if the proto-Jovian nebula did not incorporate $\mathrm{CH}_{4}$ and $\mathrm{NH}_{3}$ ices in its sateliites, the temperature may have been too high for the Jovian core to condense significant amounts of the more volatile ices. Unfortunately, this comparison is strictly applicable to the proto-Jovian and proto-Saturnian nebulae out of which the regular satellite systems formed. The temperature of the solar nebula at $5 \mathrm{AU}$ at the time of Jupiter's formation may have been colder than that prevalling during the formation of the Galilean sateliftes. In this case the composition of Ganymede and Callisto would not be representative of the material which formed Jupiter's core.

Lewis and Prinn (1980) suggested that carbon and nitrogen in the solar nebula were primarily in the form of CO and $N_{2}$. This creates an even worse problem for the accretion of volatiles onto the proto-Jovian core. because $\mathrm{CO}$ and $\mathrm{N}_{2}$ condense at even colder temperatures than 
$\mathrm{CH}_{4}$ and $\mathrm{NH}_{3}$. Carbon and $\mathrm{n} 1$ trogen would remain in the gas phase at a heliocentric distance of $5 \mathrm{AU}$. Once hydrodynamic collapse onto Jupiter's core takes place, $\mathrm{CO}$ and $\mathrm{N}_{2}$ would be converted quickly to $\mathrm{CH}_{4}$ and $\mathrm{NH}_{3}$. in the atmospheric envelope. However, this scenario predicts that the Jovian $\mathrm{C} / \mathrm{H}$ and $\mathrm{N} / \mathrm{H}$ ratios would be the same as in the solar nebula. This example 1llustrates that there are accretion models which do not lead to an enhancement in carbon and nitrogen in Jupiter's atmosphere.

Recently, Mayer and Pletzer (1986) proposed microporous amorphous $\mathrm{H}_{2} \mathrm{O}$ ice as an important component of cometary nuclei and of interstellar dust. This material adsorbs volatile gases very efficiently at low temperatures. It therefore is an attractive candidate for the icy planetesimals that accreted to form the cores of the outer planets. These authors noted that the competing mechanism for volatile retention, the formation of clathrate hydrates, may be too slow at the temperatures of the outer solar system. Thus, Jupiter's core may contain carbon and nitrogen brought in as adsorbed gases in the pores of $\mathrm{H}_{2} \mathrm{O}$ ice grains. Podolak (1977) investigated the possibility that Jupiter is globally depleted in $\mathrm{H}_{2} \mathrm{O}$. A hot proto-Jupiter could have caused $\mathrm{H}_{2} \mathrm{O}$ ice to vaporize of infalling material so that only the rocky portion was accreted into the planet. Podolak proposed a number of models of Jupiter's interior which contained large amounts of rocky materials in the outer core instead of $\mathrm{H}_{2} \mathrm{O}$. He concluded 
that a given mass of $\mathrm{H}_{2} \mathrm{O}$ in Jupiter's outer core may be replaced by an equal mass of rock and still satisfy the available gravity data for Jupiter. According to this scenar1o, Jupiter's core would have virtually none of the volatile $\mathrm{NH}_{3}$ and $\mathrm{CH}_{4}$ ices and only a small amount of $\mathrm{H}_{2} \mathrm{O}$ 1ce. Th1s might explain the observed depletion in the $0 / \mathrm{H}$ ratio in Jupiter's atmosphere if atmospheric oxygen is derlved primarily from material in the core. However, if the protoplanet were too warm to accrete a volatile-rich core, this means that the volatiles remained in the gas phase in the surrounding nebula. Once hydrodynamic collapse occured, this volatile inventory was incorporated in Jupiter's atmosphere. Once again, it is difficult to avold forming a solar composition atmosphere. Thus, reducing the volatile inventory in Jupiter's core appears to make the problem of enhanced atmospheric $\mathrm{C} / \mathrm{H}$ and $\mathrm{N} / \mathrm{H}$ even worse without solving the oxygen problem.

If we suppose that by some process Jupiter's core has a substantial amount of $\mathrm{CH}_{4}$ and $\mathrm{NH}_{3}$ ices, then there must be some communication with the outer envelope in order to enrich the observable portion of Jupiter's atmosphere in carbon and nitrogen. Stevenson (1982a, 1982b) investigated the evolution of the cores of the giant planets since their formation. He found that redistribution of layered constituents from Jupiter's outer core to the convective outer atmosphere is possible, but the process is limited by diffusion. Convection in the envelope can 
erode perhaps a few percent of the 1cy core over the age of the solar system. Stevenson inferred that the cores of the outer planets have been preserved vilutually unchanged since the formation of the solar system. He concluded that "convective dredging" is insufficient to enrich Jupiter's atmosphere in carbon by the required amount.

As an alternative, stevenson proposed that a large mass of comet-1ike planetesimals accreted onto the giant planets after formation. The composition of these infaling objects is thought to include methane clathrates which originally condensed in the outer solar system. This mechan1sm requires several Earth masses of methane clathrate in order to enrich Jupiter's atmosphere in carbon by the required amount. However, this scenario will not work if Jupiter is globally depleted in oxygen and enriched in carbon, because methane clathrates bring in about $\mathbf{s i x}$ oxygen atoms for every carbon atom (Lunine 1985).

If the efficiency of redistribution of the core is higher than Stevenson has suggested, then the nucleation model may still be appropriate. The problem now is to explain why carbon and nitrogen should be enhanced by different amounts while oxygen is depleted. This restriction is very severe and we present only some speculative 1deas here. The rate of diffusion of $\mathrm{CH}_{4}$ from the core may be higher than that of $\mathrm{H}_{2} \mathrm{O}$ due to differences in bulk properties at megabar pressure levels. The solublitty in liquid metallic hydrogen may be different for 
polar and non-polar molecules. However, this mechanism predicts that $\mathrm{NH}_{3}$ and $\mathrm{H}_{2} \mathrm{O}$ would behave similarly because both are polar molecules, whereas the observations indicate that $\mathrm{NH}_{3}$ is enhanced and $\mathrm{H}_{2} \mathrm{O}$ is depleted. Furthermore, calculations by Hubbard (private communication 1984) suggest that both carbon and oxygen are soluble at megabar pressures. Layering may have initially occurred during accretion to lock up the more refractory water ice in the inner core and the more volatile ices in the outer core. If the collapse of the massive atmospheric envelope onto the core did not change this layered structure, then perhaps $\mathrm{H}_{2} \mathrm{O}$ might remain locked up in Jupiter's core over the age of the solar system. Finally, if Podolak's models are correct, then Jupiter's core is not the source for the enrichment of volatiles in the Jovian atmosphere because the core may be composed primarily of rocky material.

In summary, the homogeneous collapse model seems incapable of explaining the observed enrichment of Jupiter's outer atmosphere in carbon and nitrogen. The nucleation model also has many problems in explaining Jupiter's gas composition. A late stage of accretion in which volatiles from the outer solar system are swept up by Jupiter may explain the observed $\mathrm{C} / \mathrm{H}$ and $\mathrm{N} / \mathrm{H}$ ratios, but this scenario cannot account for the $0 / H$ deficiency in Jupiter's atmosphere. Currently the only models which predict a global depletion in oxygen in Jupiter's atmosphere do not simultaneously explain the observed enhancement of carbon and nitrogen. 


\section{ACKNOWLEDGEMENTS}

The authors wish to thank $R$. Hanel, B. Conrath, W. Maguire, J. Pearl, R. Samuelson, M. Flasar, and J. Pirraglia of the Voyager IRIS team for their help in the analysis of the IRIS data. We also thank W. Hubbard, D. Hunten, and $J$. Lunine for helpful discussions and comments on the manuscript. We thank an anonymous referee for helpful suggestions, especially in section IV. Thanks are due to $\mathrm{J}$. Tingley and L. Mayo for their assistance with the Goddard spectrum synthesis program and $K$. Denomy for the preparation of the figures. This research was supported by NASA grant NAG2-206 and by NASA's Graduate Student Researchers Program. 
Table 1

Selection Criteria for IRIS 5 um Ensembles

\begin{tabular}{|c|c|c|c|c|c|c|}
\hline Name & $T_{5}$ & $\mathrm{~T}_{45}$ & $\theta$ & LAT & $\Delta \lambda_{\max }$ & $\Delta \lambda_{\text {min }}$ \\
\hline NEB-Hot & $>250$ & $>149$ & $<30^{\circ}$ & $5.15^{\circ}$ & $5.5^{\circ}$ & $1.2^{\circ}$ \\
\hline SEB-Hot & $>240$ & $>147$ & $<30^{\circ}$ & $-24,-6^{0}$ & $7.2^{\circ}$ & $1.6^{\circ}$ \\
\hline $\mathrm{EQZ}$ & $<230$ & $\langle 147$ & $<34^{\circ}$ & $-10,+8^{\circ}$ & $9.0^{\circ}$ & $1.2^{\circ}$ \\
\hline Cold Zones & $<210$ & $<147$ & $<45^{\circ}$ & $\pm 41^{\circ}$ & $9.5^{\circ}$ & $1.2^{\circ}$ \\
\hline Calib & --- & --- & $<30^{\circ}$ & $\pm 40^{\circ}$ & $76^{\circ}$ & $59^{\circ}$ \\
\hline
\end{tabular}


Table 2

Description of IRIS $5 \mu m^{-}$Ensembles

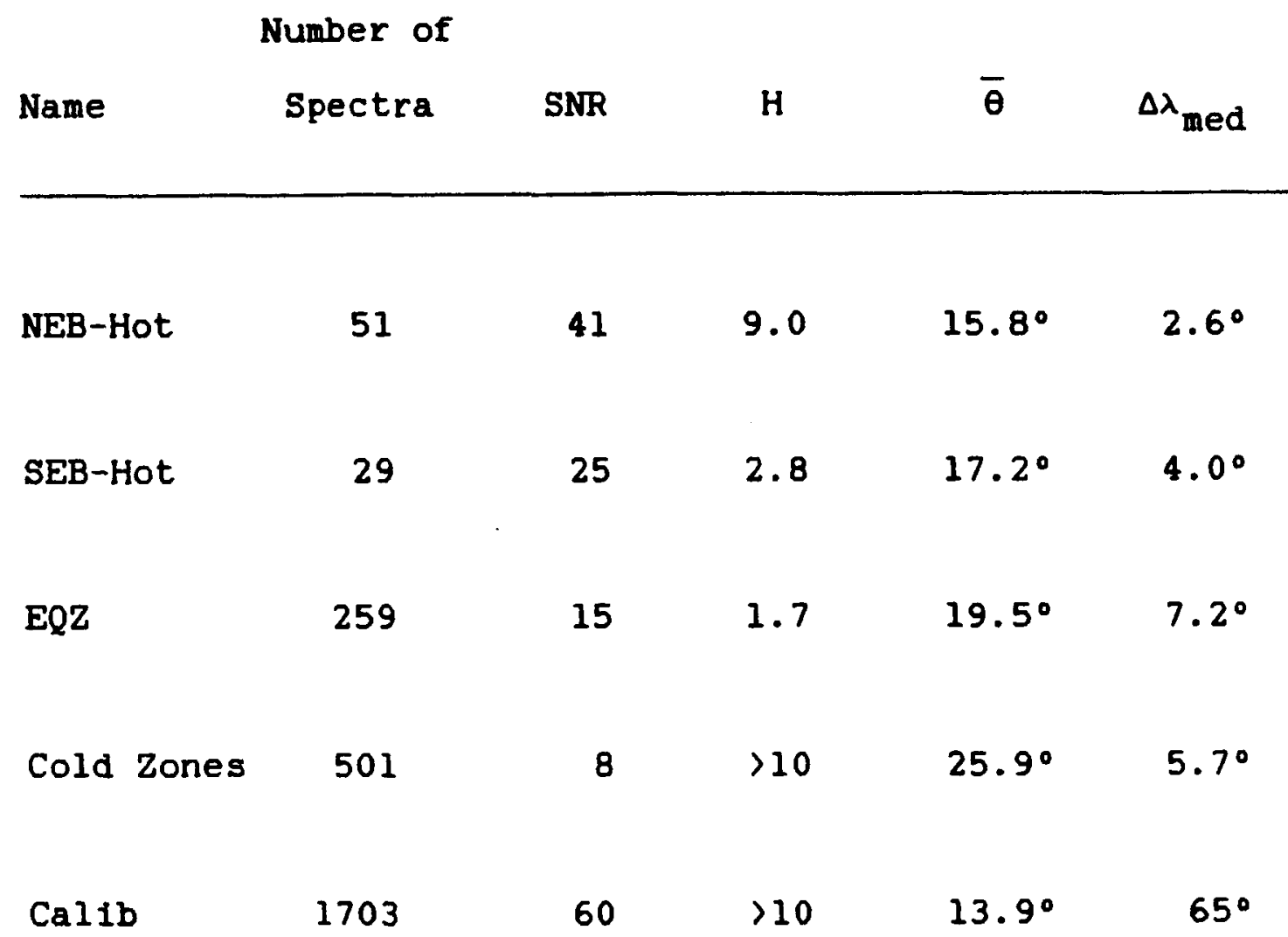




\section{REFERENCES}

Bezard, B., Baluteau, J. P., and Marten, A. 1983, Icarus, 54,434 .

Bjoraker, G. L. 1985, Ph. D. Dissertation, Univ. of Arizona.

Bjoraker, G. L., Larson, H. P., and Kunde, V. G. 1986, Icarus, vol. 66, p. 579.

Bodenheimer, P., Grossman, A. S., DeCampli, W. M., Marcy, G., and Pollack, J. B. 1980, Icarus, 41, 293.

Cameron, A. G. W. 1978, Moon and Planets, 18, 5.

DeCampli, W. M., and Cameron, A. G. W. 1979, Icarus, 38, 367.

Drossart, P., and Encrenaz, Th. 1982, Icarus, 52, 483.

Gautier, D. G., Conrath, B., Hanel R., Kunde, V., Chedin, A., and Scott, N. 1981, J. Geophys. Res., 86, 8713. 
Gaut1er, D., and Owen, T. 1983, Nature, 304, 691.

Gautier, D., and Owen, T. 1985, in Protostars and Planets II, ed. Black and Matthews, 832 .

Hanel, R., et al 1979, Science, 204, 972.

Hanel, R., et al 1980, Applied Optics, 19, 1391.

Hubbard, W. B., and Horedt, G. P. 1983, Icarus, 54, 456.

Kunde, V., et al 1982, Ap. J., 263, 443.

Kunde, V.G., and Maguire, W.C. 1974, J. Quant Spectros. Radial Transf. 14, 803-817.

Lambert, D. L. 1978, Mon. Not. Roy. Astron. Soc., 182, 249.

Larson, H. P., Davis, D. S., Hofmann, R., and Bjoraker, G. L. 1984, Icarus, 60,621 .

Larson, H. P., and Fink, U. 1975, Applied Optics, 14, 2085.

Lewis, J. S. 1969, Icarus, 10, 365.

Lewis, J. S. 1974, Science, 186, 440.

Lewis, J. S., and Prinn, R. G. 1980, Ap. J., $238,357$.

Lindal, G. F., et al 1981, J. Geophys. Res., 86, 8721. 
Lunine, J. I. 1985, Ph. D. Thes1s, California Institute of Technology.

Mayer, E., and Pletzer, R. 1986, Nature, 319, 298.

Mizuno, H. 1980, Prog. Theor. Phys., 64, 544.

Perri, F., and Cameron, A. G. W. 1974, Icarus, 22, 416.

Podolak, M. 1977, Icarus, 30, 155.

Stevenson, D. J. 1982a, Planet. Space Sc1., 30, 755.

Stevenson, D. J. 1982b, Lunar and Planet. Sci. Conf., 13, 770 .

Terrile, R. J. 1978, Ph. D. Thesis, California Institute of Technology.

Terrile, R. J., et al 1979, Science, 204, 1007.

Weidenschilling, S. J., and Lewis, J. S. 1973, Icarus, 20, 465. 
FIGURE CAPTIONS

Figure 1. Jupiter's $5 \mu \mathrm{m}$ spectrum observed from the KAO (top) is compared with the best synthetic spectrum (center) generated using a radiative transfer model. The observed spectrum is an average over all longitudes of the -40 to $40^{\circ}$ latitude region. Absorption lines of gaseous $\mathrm{H}_{2} \mathrm{O}, \mathrm{NH}_{3}, \mathrm{PH}_{3}$, $\mathrm{CH}_{4}, \mathrm{CH}_{3} \mathrm{D}, \mathrm{CO}$, and $\mathrm{GeH}_{4}$ are formed in the troposphere between 1 and 6 bars. The lower trace indicates the atmospheric pressure levels where the gas optical depth equals unity. The maximum contribution to the emergent radiation originates from this level. Vertical bars identify the positions of $21 \mathrm{H}_{2} \mathrm{O}$ absorption features discussed in the text as well as the pressure levels where they are formed in Jupiter's atmosphere.

Figure 2. Four vertical distributions of Jovian $\mathrm{H}_{2} \mathrm{O}$ are shown as functions of pressure (left axis) and temperature (right axis). These are used to calculate synthetic spectra for comparison with the KAO data. Profile 3 provides the best fit.

Figure 3. Synthetic spectra of Jupiter calculated using $\mathrm{H}_{2} \mathrm{O}$ Profiles 1,3 , and 4 of Fig. 2 are indicated by traces 
a), b), and c), respectively. Line opacity is due to $\mathrm{H}_{2} \mathrm{O}$ alone: continuum opacity is due to $\mathrm{H}_{2}$ and to an absorbing cloud. Each spectrum is normalized at $2130 \mathrm{~cm}^{-1}$. Arrows denote strong, medium, and weak $\mathrm{H}_{2} \mathrm{O}$ lines discussed in the text.

Figure 4. A comparison of Jupiter's observed spectrum from the KAO with 3 synthetic spectra for $v=1962$ to $2002 \mathrm{~cm}^{-1}$. The synthetic spectra include all known $5 \mu \mathrm{m}$ absorbers. The spectra are calculated using 3 of the $\mathrm{H}_{2} \mathrm{O}$ profiles defined in Fig. 2. Arrows and numbers identify $\mathrm{H}_{2} \mathrm{O}$ lines; the strength category (strong, medium, or weak) is displayed under each number. The $\mathrm{PH}_{3}$ absorption feature at $1972 \mathrm{~cm}^{-1}$ is discussed in the text.

Figure 5. Same as Fig. 4 but for $v=2063$ to $2093 \mathrm{~cm}^{-1}$. The synthetic spectra are calculated using $\mathrm{H}_{2} \mathrm{O}$ Profiles 2 . 3 , and 4 .

Figure 6. Same as Fig. 4 but for $v=2120$ to $2150 \mathrm{~cm}^{-1}$. The synthetic spectra calculated using $\mathrm{H}_{2} \mathrm{O}$ Profiles 3 and 4 are compared with the observed Jupiter spectrum.

Figure 7. Voyager IRIS spectra of Jovian Hot Spots in the North (a) and South (b) Equatorial Belts are compared with best-fit synthetic spectra. Instrumental error bars are displayed. Arrows denote $\mathrm{H}_{2} \mathrm{O}$ lines. The integrated column 
abundance of $\mathrm{H}_{2} \mathrm{O}$ above the 4 bar level is indicated for each spectrum.

Figure 8. Same as Fig. 7 but for IRIS spectral averages of the Equatorial zone (a) and for zones exhibiting the coldest $5 \mu \mathrm{m}$ temperatures (b).

Figure 9. Four vertical distributions of Jovian $\mathrm{H}_{2} \mathrm{O}$ are shown as functions of pressure and temperature. They are used to calculate synthetic spectra for comparison with IRIS $5 \mu \mathrm{m}$ observations. Profiles 1 and 4 are from Fig. 2. Profile NEB fits the spectra of the NEB Hot Spots. Profile Zone matches the IRIS spectra of the zones and SEB Hot Spots.

Figure 10. Voyager IRIS observations of the Hot Spots in the NEB (trace a) are compared with those in the SEB (b). The $\mathrm{H}_{2} \mathrm{O}$ lines in the SEB are stronger than in the NEB. The radiance scales are chosen so that the continuum levels coincide for the two spectra.

Figure 11. The vertical distribution of $\mathrm{H}_{2} \mathrm{O}$ in Jupiter's troposphere is displayed for the NEB Hot spots, zones, and for an average between -40 and $40^{\circ}$ latitude. The pressure levels where $\mathrm{H}_{2} \mathrm{O}$ lines are formed are indicated for each of the 3 strength categories. Vertical bars indicate the pressure range over which $\mathrm{H}_{2} \mathrm{O}$ lines are formed; horizontal bars denote the uncertainty in $\mathrm{H}_{2} \mathrm{O}$ mole fraction 
at each level. Our $\mathrm{H}_{2} \mathrm{O}$ abundance is substantially smaller than the solar composition model (dashed line). The base of the $\mathrm{H}_{2} \mathrm{O}$ ice cloud is determined by the level where the $\mathrm{H}_{2} \mathrm{O}$ gas abundance curve and saturated vapor pressure curves intersect. Our measurements imply that the $\mathrm{H}_{2} \mathrm{O}$ cloud $1 \mathrm{~s}$ at 2 bars, rather than at the 5 bar predicted level. 
ADDRESSES

GORDON L. BJORAKER and VIRGIL G. KUNDE: Code 693.2, NASA Goddard Space

Flight Center,Greenbelt, MD 20771.

HAROLD P. LARSON : Lunar and Planetary Laboratory, University of

Arizona, Tucson, AZ 85721. 


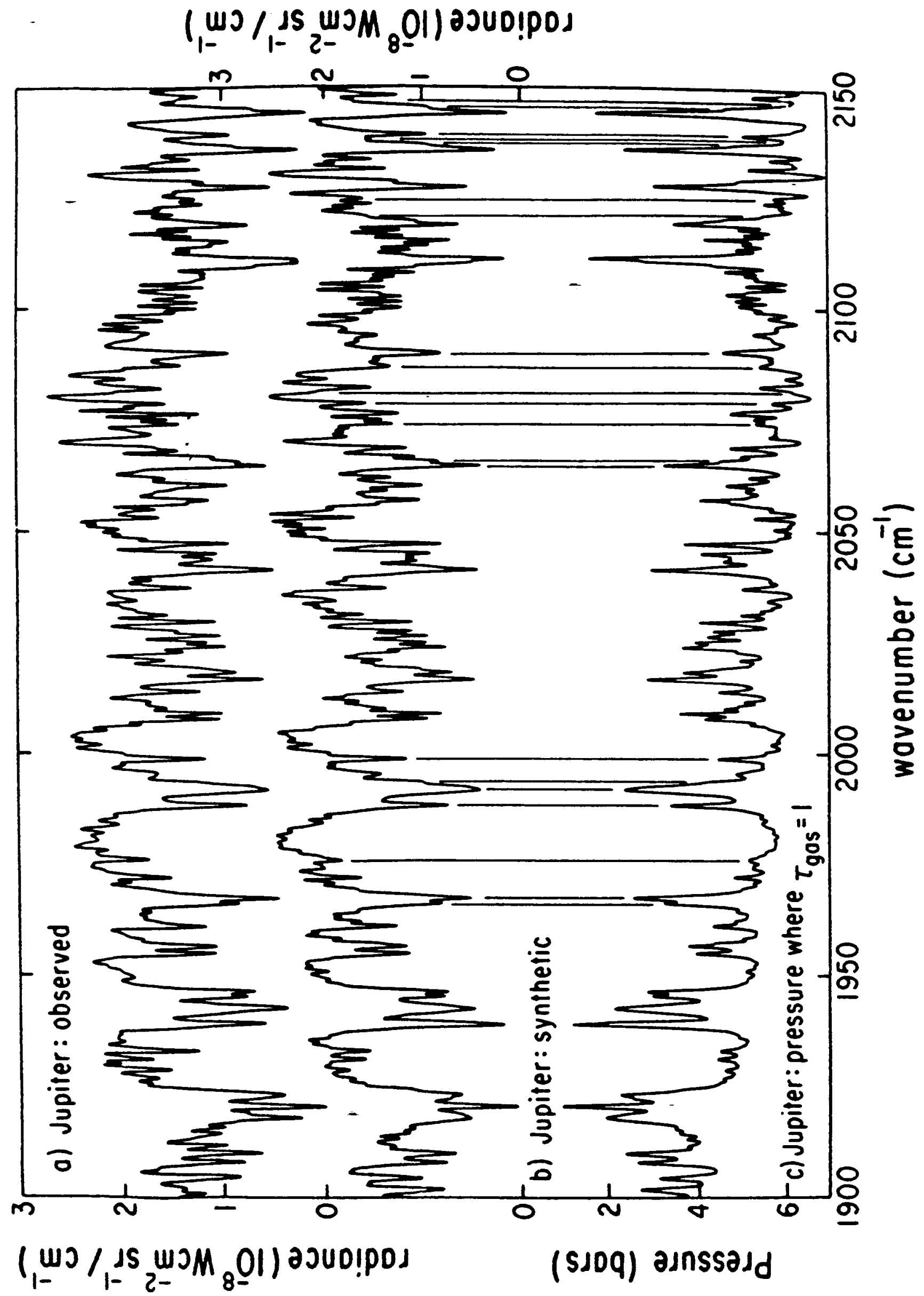




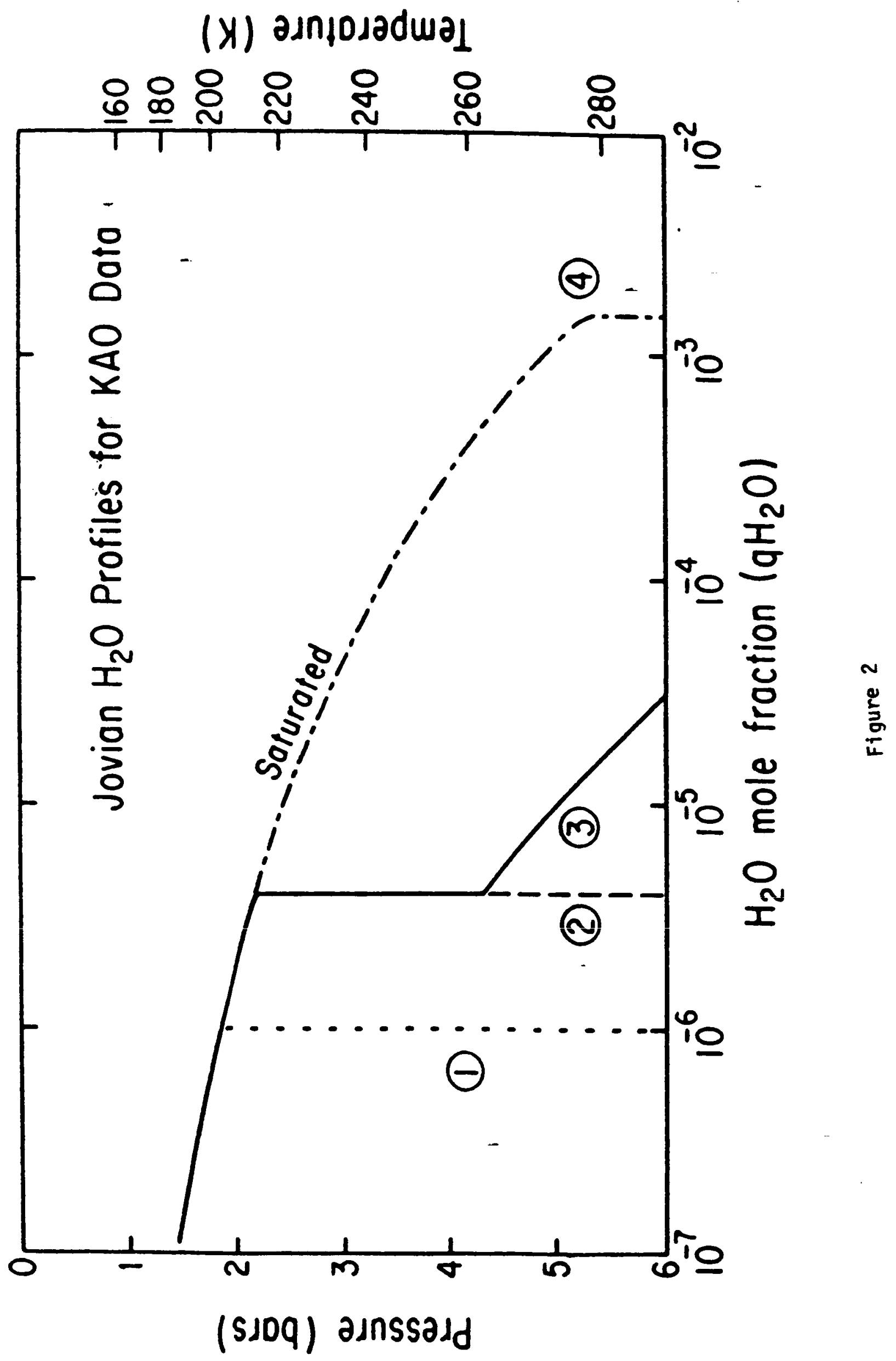




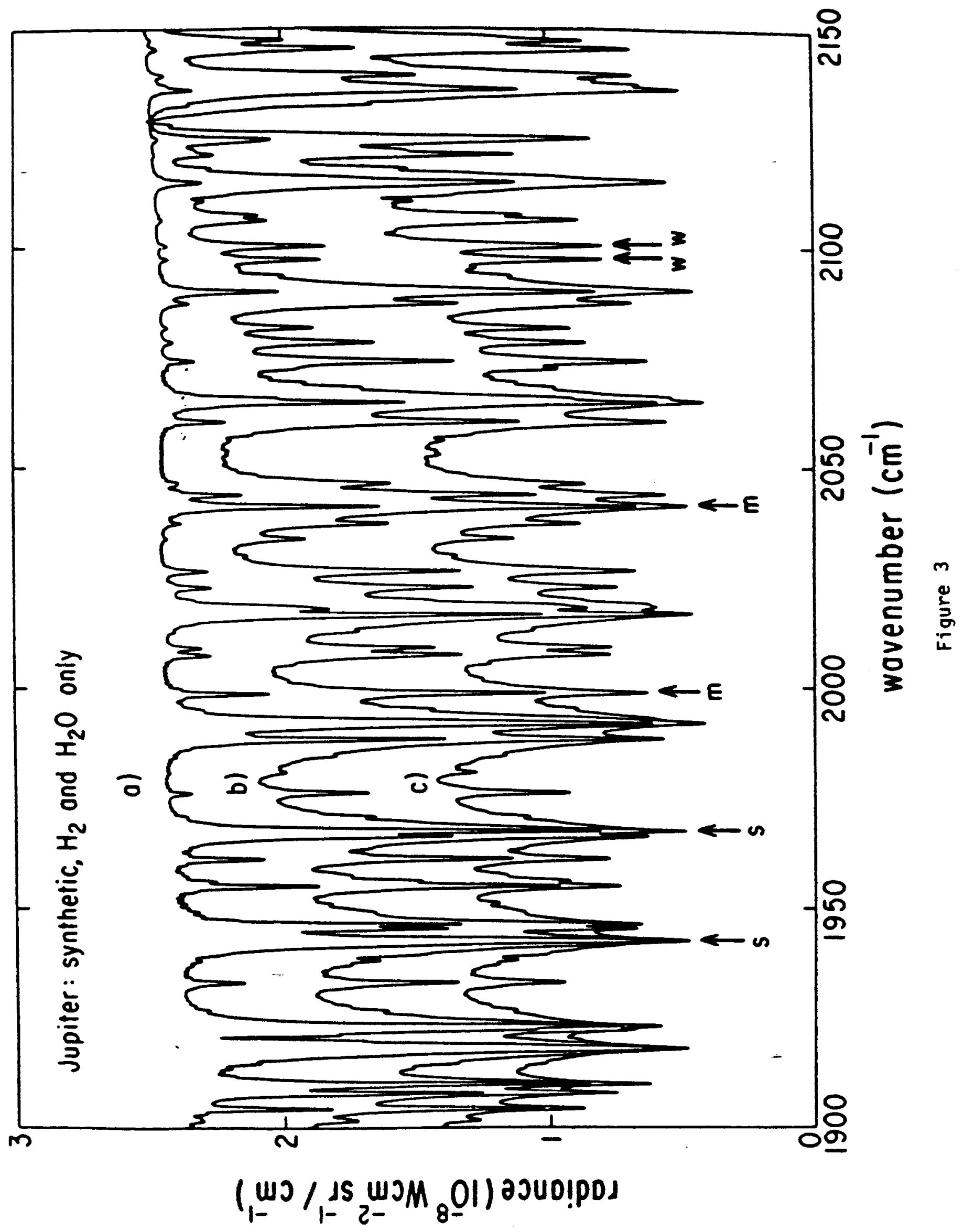




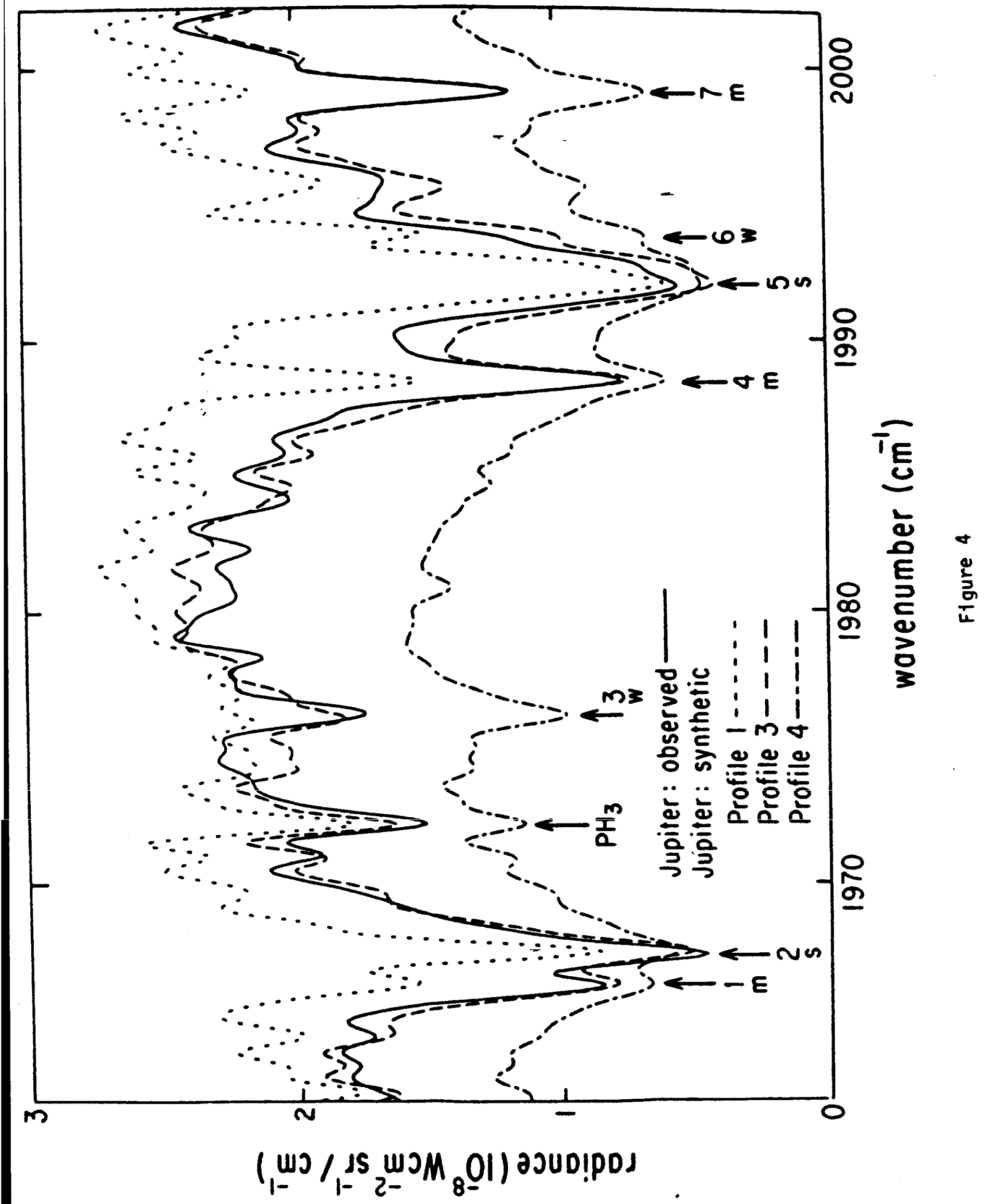




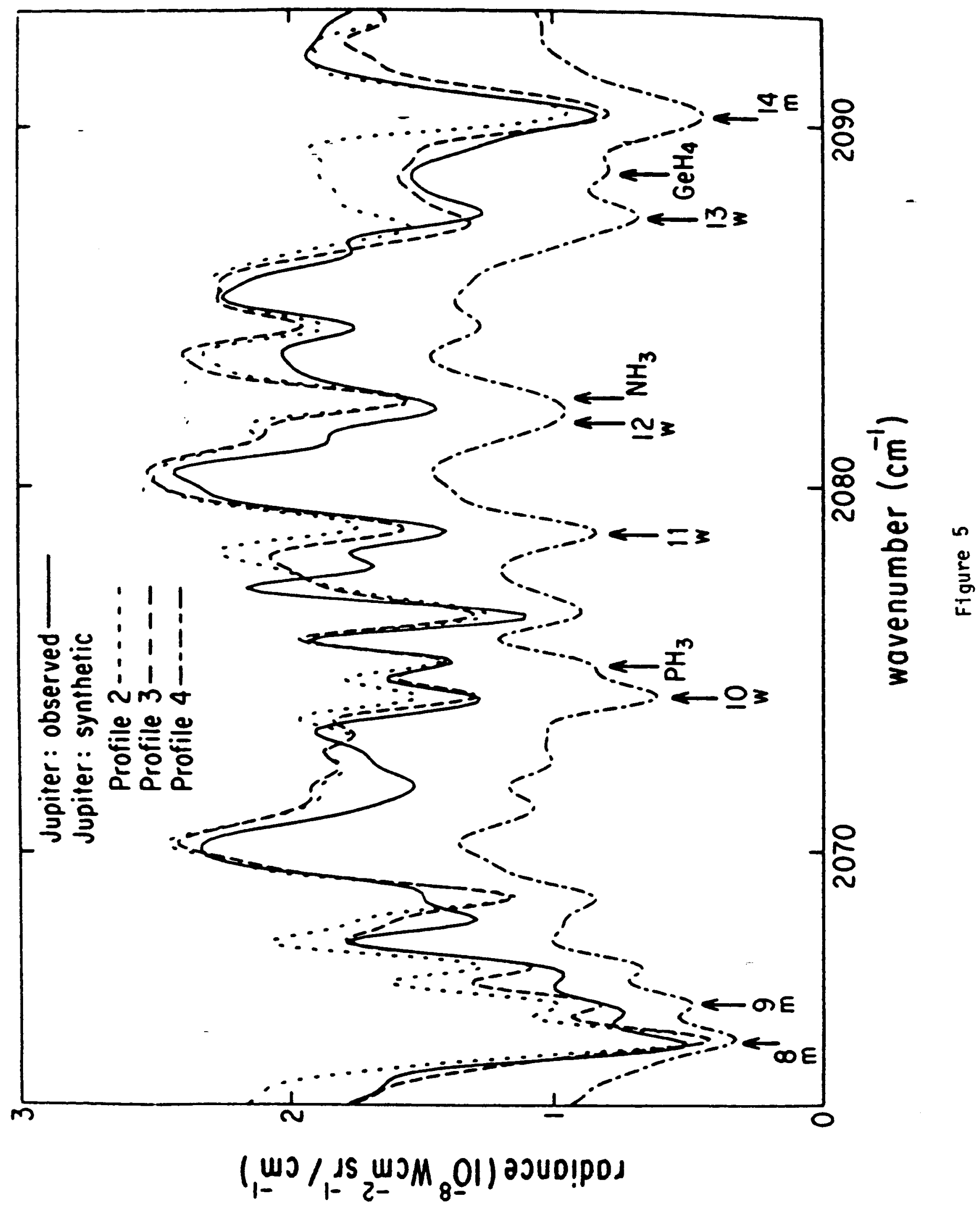




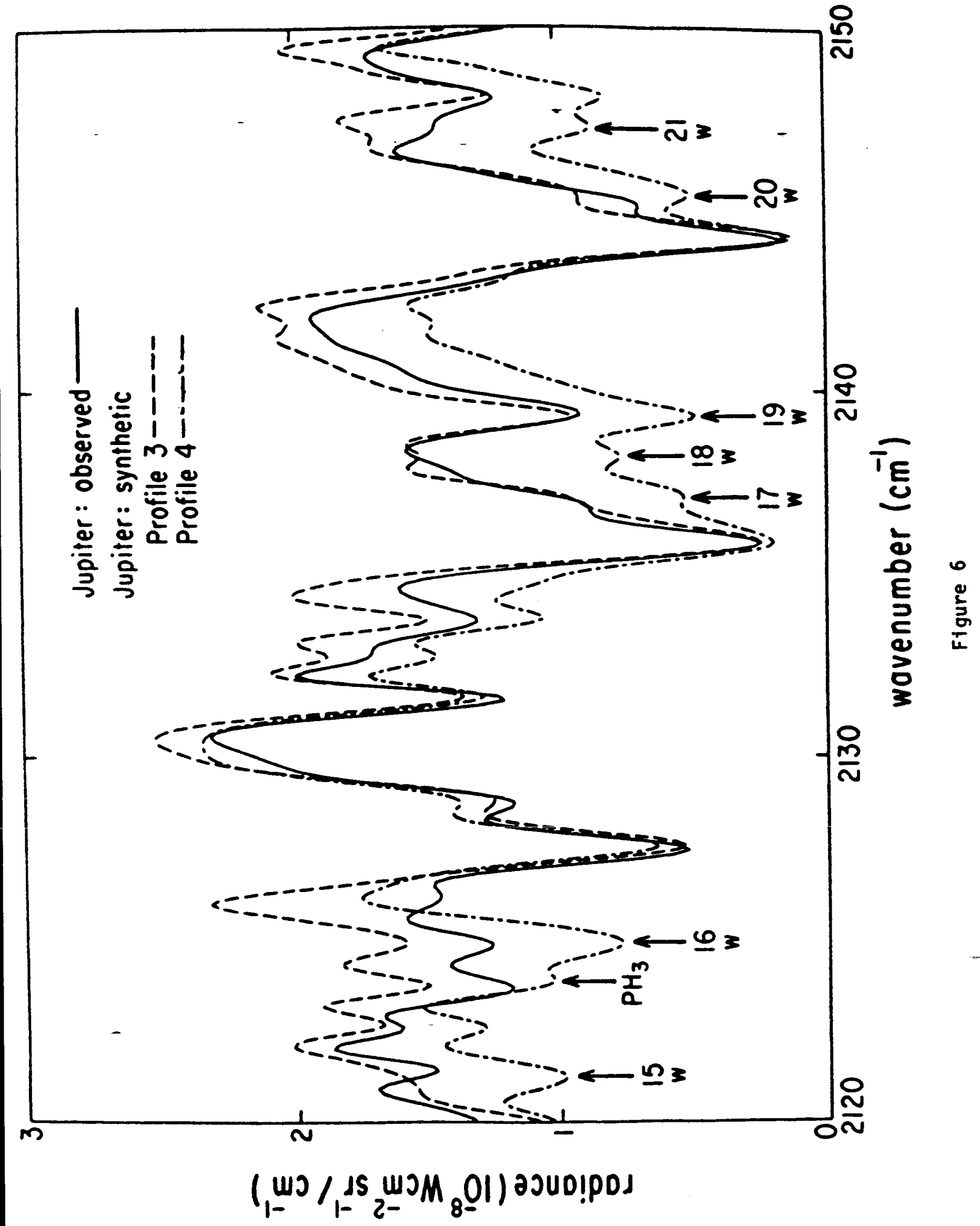




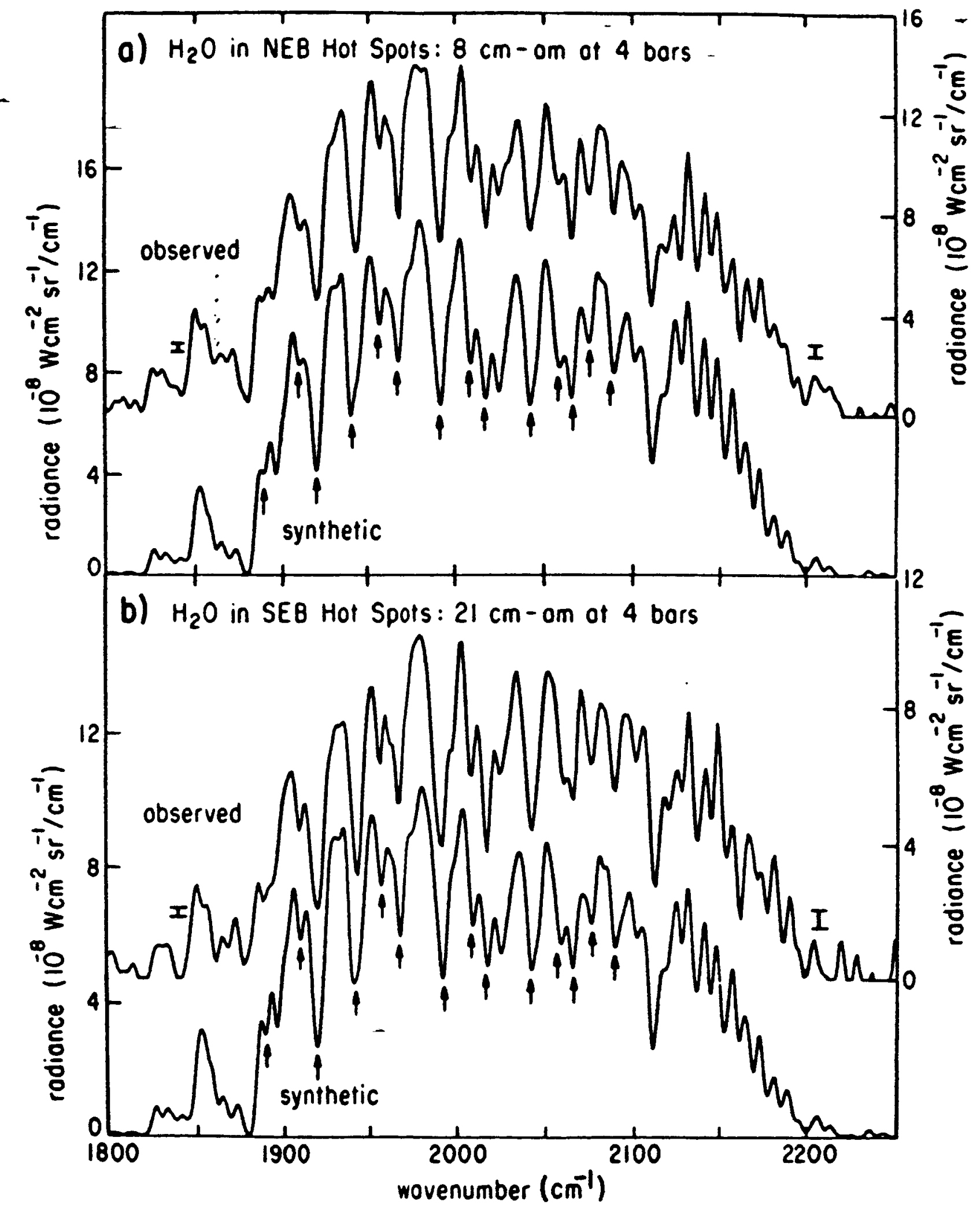

Figure 7 


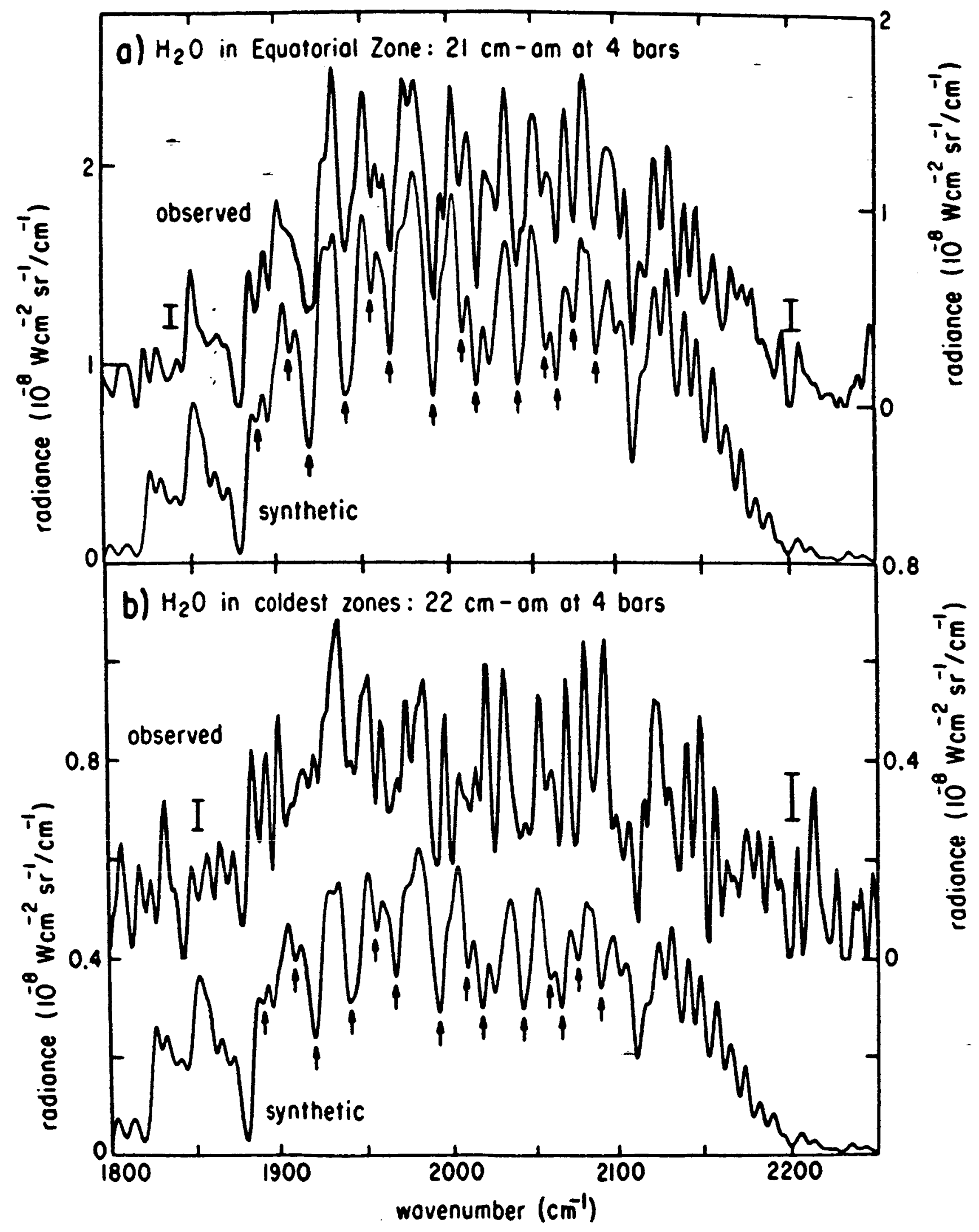

Figure 8 


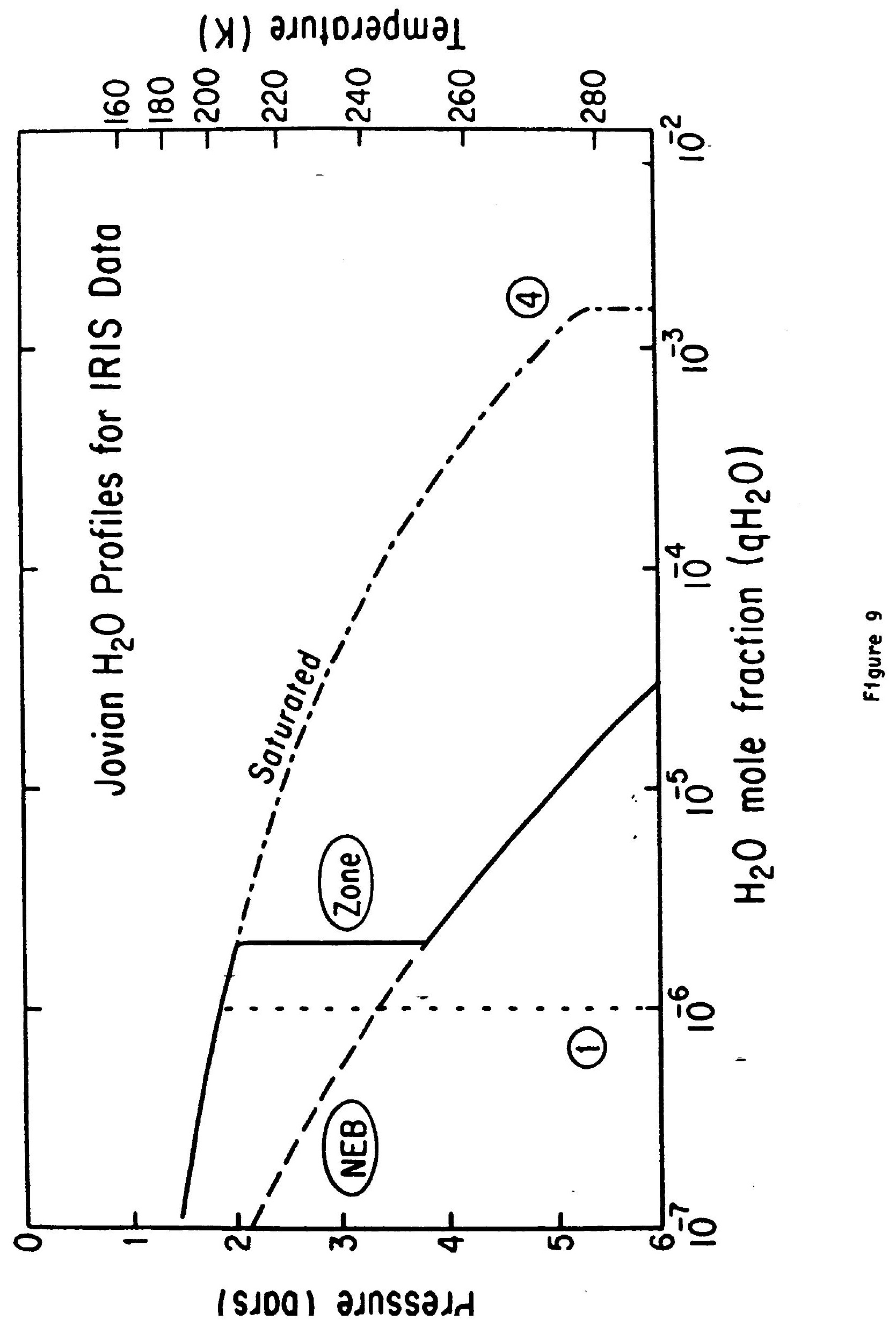




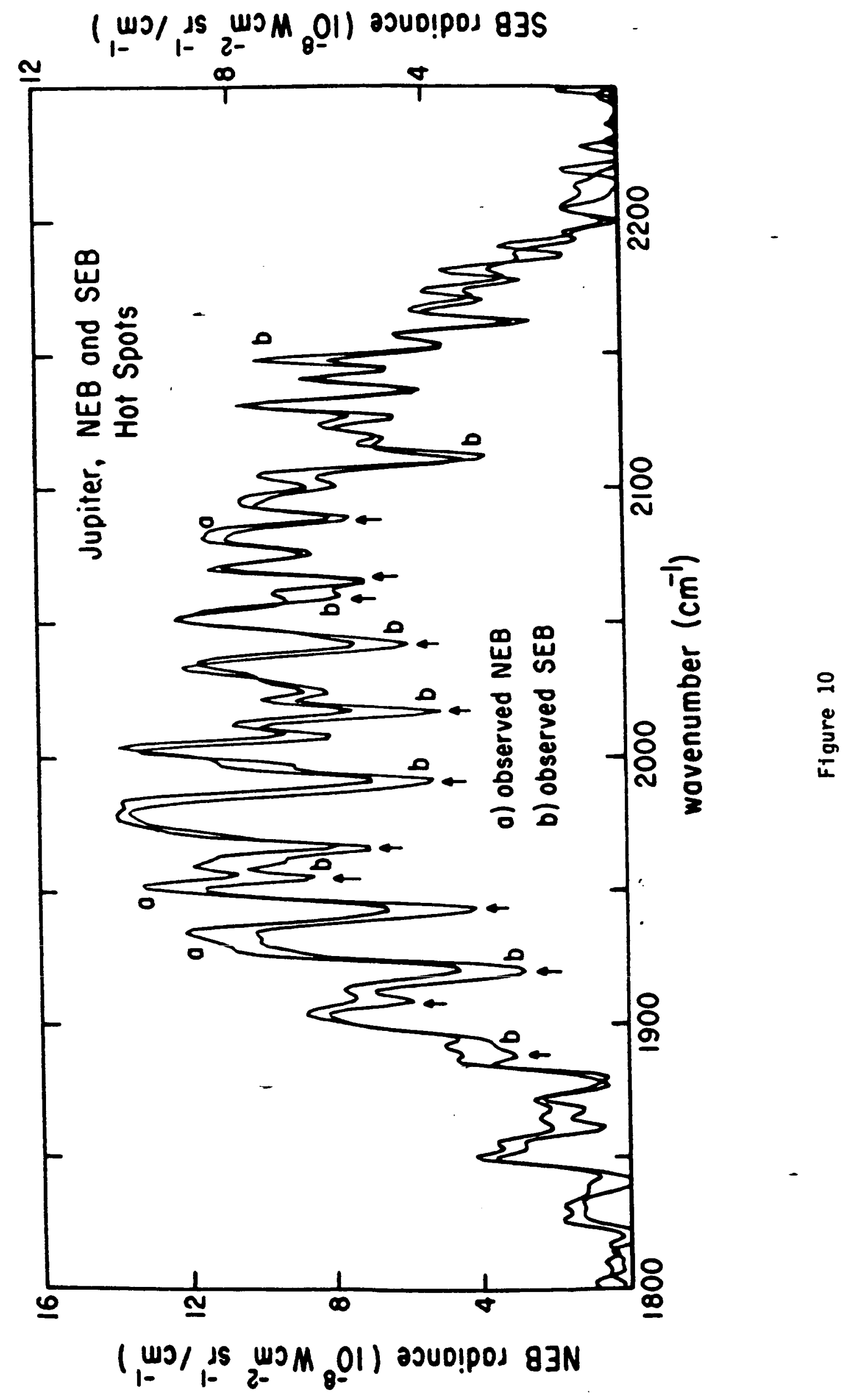




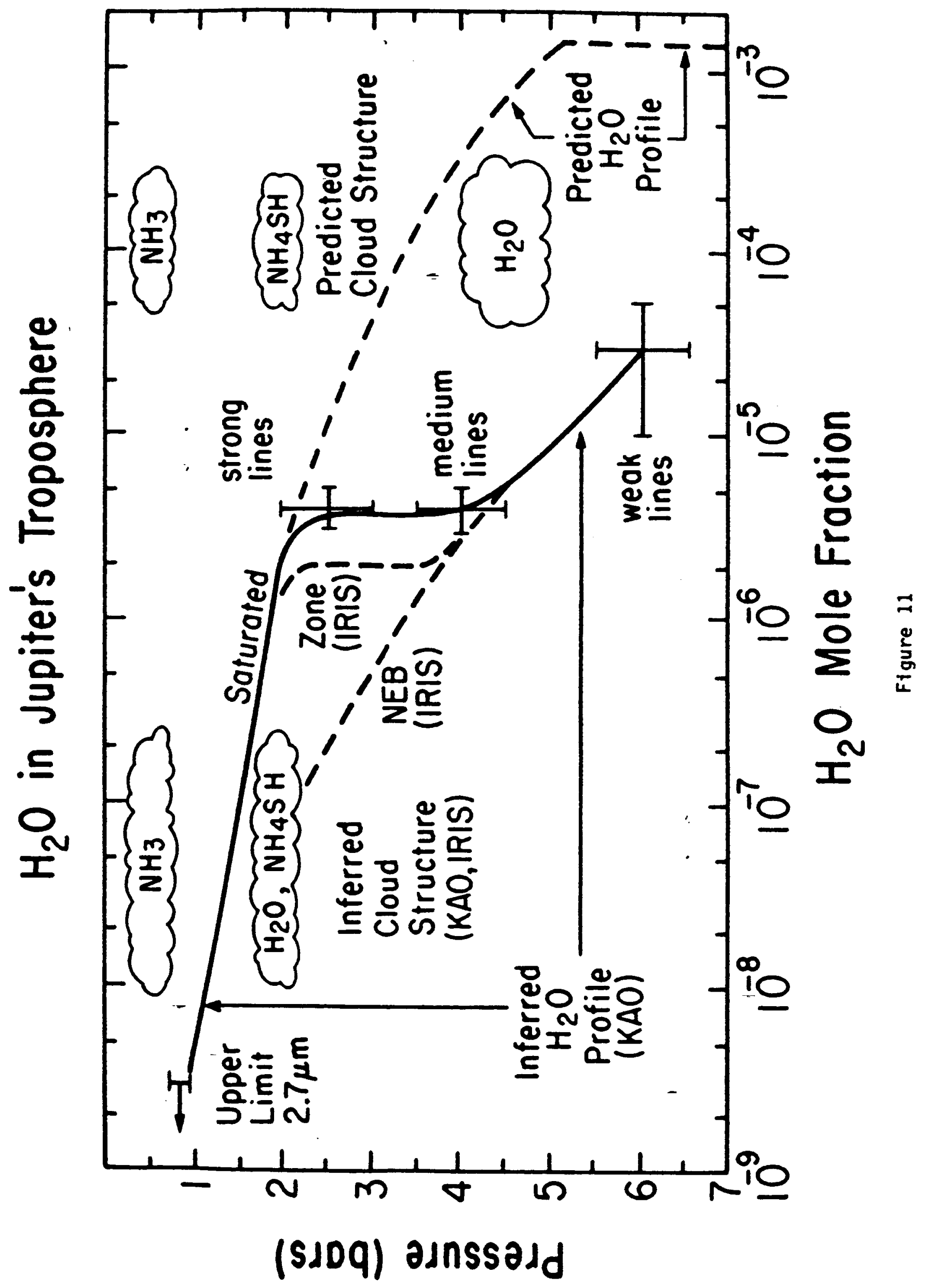




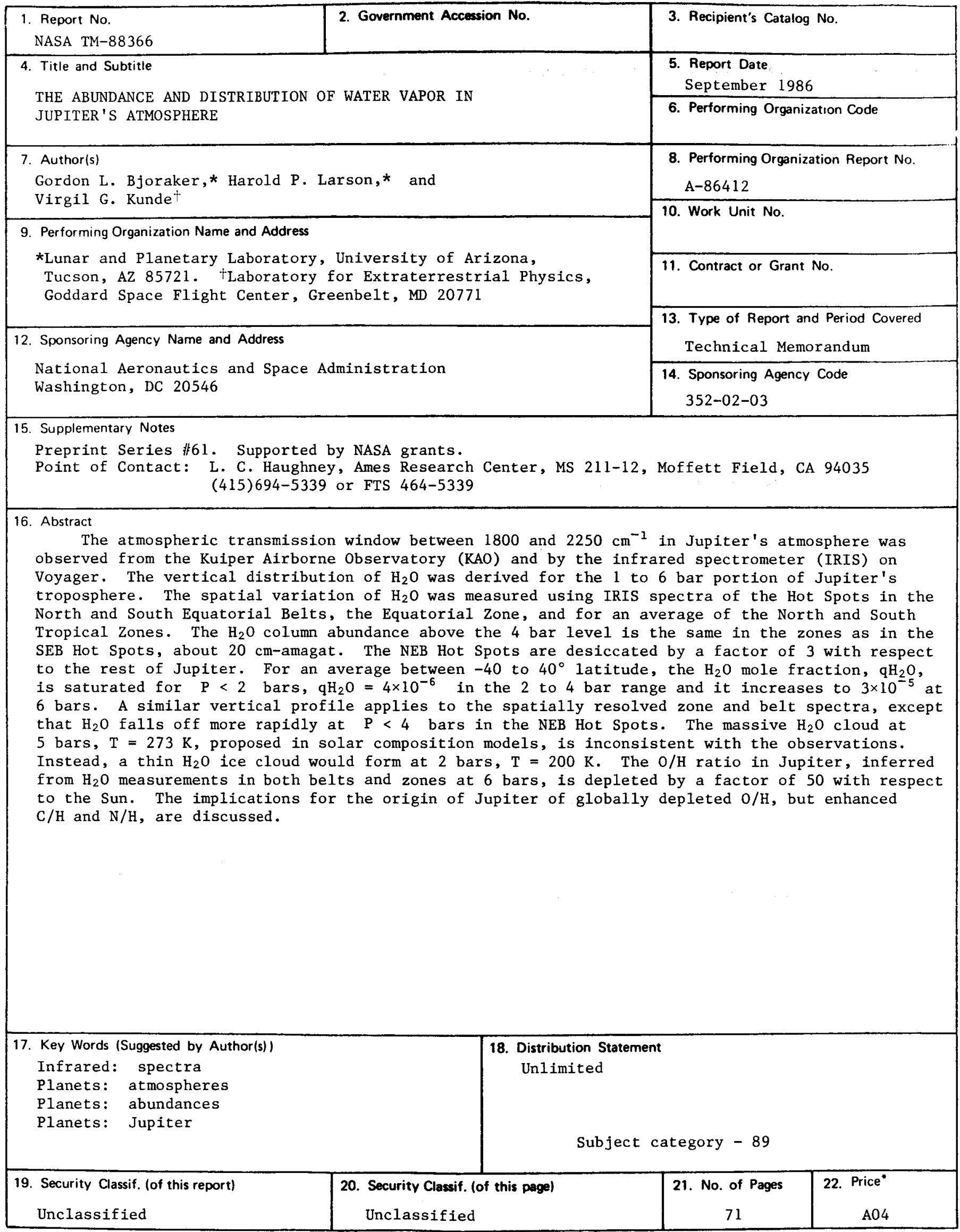

"For sale by the National Technical Information Service, Springfield, Virginia 22161 